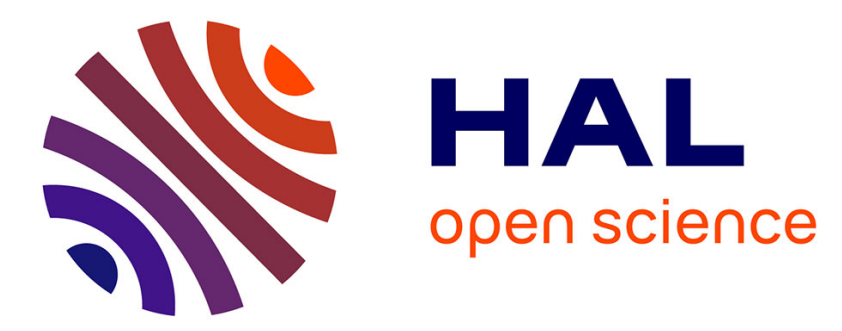

\title{
Exact exponent for the number of persistent spins in the zero-temperature dynamics of the one-dimensional Potts model
}

\author{
Bernard Derrida, Vincent Hakim, Vincent Pasquier
}

\section{- To cite this version:}

Bernard Derrida, Vincent Hakim, Vincent Pasquier. Exact exponent for the number of persistent spins in the zero-temperature dynamics of the one-dimensional Potts model. Journal of Statistical Physics, 1996, 85, pp.763-797. 10.1007/BF02199362 . hal-03282969

\section{HAL Id: hal-03282969 \\ https://hal.science/hal-03282969}

Submitted on 19 Jul 2021

HAL is a multi-disciplinary open access archive for the deposit and dissemination of scientific research documents, whether they are published or not. The documents may come from teaching and research institutions in France or abroad, or from public or private research centers.
L'archive ouverte pluridisciplinaire HAL, est destinée au dépôt et à la diffusion de documents scientifiques de niveau recherche, publiés ou non, émanant des établissements d'enseignement et de recherche français ou étrangers, des laboratoires publics ou privés. 


\title{
Exact Exponent for the Number of Persistent Spins in the Zero-Temperature Dynamics of the One-Dimensional Potts Model
}

\author{
Bernard Derrida, ${ }^{1,2}$ Vincent Hakim, ${ }^{1}$ and Vincent Pasquier ${ }^{2}$
}

\author{
Received January 30, 1996
}

For the zero-temperature Glauber dynamics of the $q$-state Potts model, the fraction $r(q, t)$ of spins which never flip up to time $t$ decays like a power law $r(q, t) \sim t^{-\theta(q)}$ when the initial condition is random. By mapping the problem onto an exactly soluble one-species coagulation model $(A+A \rightarrow A)$ or alternatively by transforming the problem into a free-fermion model, we obtain the exact expression of $\theta(q)$ for all values of $q$. The exponent $\theta(q)$ is in general irrational, $\theta(3)=0.53795082 \ldots, \theta(4)=0.63151575 \ldots, \ldots$, with the exception of $q=2$ and $q=\infty$, for which $\theta(2)=3 / 8$ and $\theta(\infty)=1$.

KEY WORDS: Glauber dynamics; coarsening: free fermions; Toeplitz determinant; Potts model; reaction-diffusion problems.

\section{INTRODUCTION}

One of the simplest systems for which coarsening(1) associated to phase ordering can be studied is the one-dimensional Ising or $q$-state Potts model evolving according to zero-temperature Glauber dynamics. ${ }^{(2)}$ At zero temperature, the evolution tends to align all the spins. Consequently, for a random initial condition, one observes domains of parallel spins growing with time.

One way to implement the zero-temperature dynamics of these onedimensional systems is to update every spin during each infinitesimal time interval $\Delta t$ according to the following dynamics:

\footnotetext{
' Laboratoire de Physique Statistique, ENS, F-75231 Paris Cedex 05. France.

'Service de Physique Théorique, CE Saclay, F-91191 Gif sur Yvette, France.
} 


$$
\begin{aligned}
S_{i}(t+\Delta t) & =S_{i}(t) & \text { with probability } & 1-2 \Delta t \\
& =S_{i-1}(t) & \text { with probability } & \Delta t \\
& =S_{i+1}(t) & \text { with probability } & \Delta t
\end{aligned}
$$

Therefore, updating a spin in one dimension consists in choosing for its new value the value of one of its two neighbors at random. When the value $S_{i}(t)$ of the spin $i$ at time $t$ is traced back in time, a random walk is obtained which connects site $i$ through various ancestors to a particular site $i_{0}$ in the initial configuration, so that $S_{i}(t)=S_{i, 1}(0)$. The dynamics is nothing but the dynamics of a voter model ${ }^{(3)}$ and this makes many properties computable via random walk methods.

For example, the equal-time correlation function $\Phi_{i}(i, j)=\left\langle\delta_{S_{i}(t) . S_{j}(t)}\right\rangle$ evolves according to

$$
\begin{aligned}
\frac{d \Phi_{r}(i, j)}{d t}= & \Phi_{l}(i+1, j)+\Phi_{r}(i-1, j)+\Phi_{r}(i, j+1) \\
& +\Phi_{l}(i, j-1)-4 \Phi_{l}(i, j)
\end{aligned}
$$

For a random initial condition, $\Phi_{0}(i, j)=1 / q+[(q-1) / q] \delta_{i, j}$ and it is easy to verify that

$$
\Phi_{I}(i, j)=1-\frac{q-1}{q} C_{I}(i, j)
$$

where $C_{t}(i, j)$ is the probability that two random walkers starting at sites $i$ and $j$ at $t=0$ do not meet up to time $t$ [according to (2), during each infinitesimal time interval $\Delta t$, each walker hops to its right with probability $\Delta t$, to its left with probability $\Delta t$, and does not move with probability 1-2 $\Delta t]$ ]. Indeed, $C_{l}(i, j)$ satisfies the same equation (2) as $\Phi_{l}$ with $C_{0}(i, j)=1-\delta_{i, j}$. The solution is, for $i<j$,

$$
C_{,}(i, j)=\frac{1}{2 \pi} \int_{-\pi}^{\pi} \frac{\sin \theta \sin [(j-i) \theta]}{1-\cos \theta} e^{-4(1-\cos \theta) !} d \theta
$$

For large $t, C_{t}(i, j)$ takes a scaling form

$$
C_{l}(i, j) \simeq \frac{1}{\sqrt{2 \pi}} \int_{0}^{|i-j| / \sqrt{t}} e^{-u^{2} / 8} d u
$$

and one finds that the size of the domains increases like $t^{1 / 2}$ as expected when the order parameter is not conserved. One recovers in that way the exact scaling form (3), (4) of the equal-time pair correlation function. ${ }^{(4-8)}$ 
Random walks can also be used to calculate the autocorrelation function. From the definition of the dynamics (1), one can relate the autocorrelation to the probability that a random walk returns to its starting point at time $t$ and show that

$$
\left\langle\delta_{S_{i}(t), S_{i}(0)}\right\rangle-\frac{1}{q}=\frac{q-1}{q} \frac{1}{2 \pi} \int_{-\pi}^{\pi} d \theta e^{-2 t(1-\cos \theta)} \simeq \frac{q-1}{q} \frac{1}{2 \sqrt{\pi t}}
$$

and this gives the exponent characterizing the decay of the autocorrelation function. ${ }^{(9-11)}$

From these two well-known facts ${ }^{(3)-(5)}$ one could believe that all the exponents appearing in the zero-temperature growth of domains in one dimension are simple and well understood. However, by studying the fraction of spins $r(q, t)$ which have never flipped up to time $t$, it was found, first numerically ${ }^{(12-14)}$ and then analytically, ${ }^{115)}$ that this fraction $r(q, t)$ of persistent spins decreases with time like a power law

$$
r(q, t) \sim t^{-\theta(q)}
$$

where the exponent $\theta(q)$ has a complicated $q$ dependence. In an earlier letter $^{(15)}$ we gave for the $1 \mathrm{D}$ Potts model the exact expression of $\theta(q)$,

$$
\theta(q)=-\frac{1}{8}+\frac{2}{\pi^{2}}\left[\cos ^{-1}\left(\frac{2-q}{\sqrt{2} q}\right)\right]^{2}
$$

which agrees with all the previous numerical estimates based on Monte Carlo simulations and finite-size scaling. ${ }^{(12-14)}$ Our derivation of (7) in ref. 15 was based on a mapping onto a reaction-diffusion model $(A+A \rightarrow A)$ with a fixed source, the steady state of which was solved exactly for finite lattices. The expression of $\theta(q)$ was then obtained from a finite-size scaling argument.

Here we work directly on the infinite system and we obtain the exact expression of $r(q, t)$ valid at all times. The main advantage is that we avoid using finite-size scaling and the asymptotics become easier to analyze.

The paper is organized as follows. In Section 2, we recall the relation between the coarsening problem and the reaction-diffusion problem. ${ }^{(16-20)}$ In Section 3 we calculate for the semiinfinite chain the probability $A_{N}\left(\tau_{1}, \tau_{2}, \ldots, \tau_{N}\right)$ that the spin at the origin takes the same value at time $\tau_{1}, \tau_{2}, \ldots, \tau_{N}$. In Section 4, we take the continuous limit of the expression $A_{N}\left(\tau_{1}, \ldots, \tau_{N}\right)$ and obtain the exact expression of the fraction $r(q, t)$ of spins 
which never flip up to time $t$ for an infinite chain. In Section 5, we extract the exponent $\theta(q)$ from the asymptotic behavior of $r(q, t)$. In Section 6, we recall the results of our previous work ${ }^{(15)}$ for the finite geometry and we show in Section 7 how they can be recovered by a fermionic approach.

\section{RELATION TO A REACTION-DIFFUSION MODEL}

In the introduction we already mentioned the close connection between the zero-temperature dynamics of the Potts model and random walks. What (1) tells us is that to know the value of a spin $S_{i}(t)$ at time $t$, one needs to look at a random walker starting at time 0 from site $i$ [this random walker has the hopping rates given by $(1)]$. If this random walker is on site $i_{0}$ at time $t$, then $S_{i}(t)=S_{i_{0}}(0)$.

It is important to notice that when we relate the evolution of the spins and of the random walkers, we have to reverse the arrow of time.

To compare the value of two spins $S_{i}(t)$ and $S_{j}(t)$ at time $t$, one needs to consider two random walkers, one starting at site $i$ and the other one at site $j$. After time $t$, either the two walkers have merged into one walker which ends up on site $k_{0}$ at time $t$ and consequently $S_{i}(t)=S_{j}(t)=S_{k_{0}}(0)$, or the two walkers have not met and end up at time $t$ on sites $i_{0}$ and $j_{0}$ and consequently $S_{i}(t)=S_{i_{0}}(0)$ and $S_{j}(t)=S_{j_{0}}(0)$. This simple connection with coalescing random walks is both the origin of expression (3) and the basis $^{(15)}$ of our approach to calculating $r(q, t)$.

Let us now compare the values of the spin at the origin at two different times $\tau_{1}$ and $\tau_{2}$ where $0<\tau_{1}<\tau_{2}<t$ : one has to study two random walkers starting at the origin at times $t-\tau_{2}$ and $t-\tau_{1}$. If the two walkers merge before time $t$ and the new walker ends up at site $k_{0}$, one has $S_{0}\left(\tau_{1}\right)=S_{0}\left(\tau_{2}\right)=S_{k_{0}}(0)$. On the contrary, if the two walkers do not meet before time $t$ and end up at positions $i_{0}$ and $j_{0}$, then $S_{0}\left(\tau_{1}\right)=S_{i_{0}}(0)$ and $S_{0}\left(\tau_{2}\right)=S_{j_{0}}(0)$. One can repeat this reasoning to compare the value of the spin at the origin at $N$ different times $0<\tau_{1}<\tau_{2}<\cdots \tau_{N}<t$. One has to consider $N$ coalescing random walkers starting from the origin at times $t-\tau_{N}, t-\tau_{N-1}, \ldots, t-\tau_{1}$. At time $t$ there is a probability $P(m, t)$ that there are exactly $m$ walkers left in the system. Then the probability that $S_{0}\left(\tau_{1}\right)=$ $S_{0}\left(\tau_{2}\right)=\cdots S_{0}\left(\tau_{N}\right)$ is given by

$$
\operatorname{Prob}\left\{S\left(\tau_{1}\right)=S\left(\tau_{2}\right)=\cdots S\left(\tau_{N}\right)\right\}=\sum_{m=1}^{N} P(m, t) \frac{1}{q^{m-1}}
$$

as when the walkers occupy $m$ sites at time $t$, the probability that the spins of these $m$ sites have the same color is $1 / q^{m-1}$. Taking the limit $N \rightarrow \infty$ and 
a dense set of times $\tau_{1}, \tau_{2}, \ldots, \tau_{N}$ between 0 and $t$, one obtains the probability $r(q, t)$ that the spin at the origin never flips up to time $t$.

The above reasoning is valid for the spin at the origin of an infinite chain. It is also valid for other one-dimensional geometries (or would be valid for the voter model in higher dimension). It turns out that it is more convenient to use a semiinfinite chain $(0 \leqslant i)$, where the origin is also the site at the boundary and is updated according to

$$
\begin{array}{rlrl}
S_{0}(t+\Delta t) & =S_{0}(t) \quad \text { with probability } & 1-\Delta t \\
& =S_{1}(t) \quad \text { with probability } \quad \Delta t
\end{array}
$$

In Section 4 , we derive an exact expression for the probability $R\left(q ; t_{1}, t_{2}\right)$ that the spin at the origin of a semiinfinite chain never flips between times $t_{1}$ and $t_{2}$. The knowledge of $R\left(q ; t_{1}, t_{2}\right)$ leads to the probability $r(q, t)$ that a spin of an infinite chain never flips up to time $t$,

$$
r(q, t)=[R(q ; 0, t)]^{2}
$$

because for an infinite chain, if the spin at the origin never flips, the two sides of the origin are completely decoupled and domain walls at the right and at the left of the origin move and react with no correlation between the right and the left.

The semiinfinite chain is simpler than the infinite chain because when $N$ random walkers start from the origin at times $t-\tau_{N}<t-\tau_{N-1} \ldots$ $<t-\tau_{1}$ on a semiinfinite chain (when a walker is at the origin, it can only hop to its right), they remain always in the same order, corresponding to their ages: at time $t$ one always has $i_{1} \leqslant i_{2} \leqslant \cdots i_{N}$. This fact makes easier the calculation of the probability that no pair (among these $N$ walkers) has met until time $t$ : if $c_{i, j}$ is the probability that two walkers (on the semiinfinite chain) starting at times $t-\tau_{i}>t-\tau_{j}$ do not meet up to time $t$, one can show that the probability $c_{i_{1}, i_{2}, i_{3}, i_{4}}^{(2)}$ that no pair meets (up to time $t$ ) among four walkers starting at times $t-\tau_{i_{1}}>t-\tau_{i_{2}}>t-\tau_{i_{3}}>t-\tau_{i_{4}}$ (from the origin) is given by

$$
c_{i_{1}, i_{2}, i_{3}, i_{4}}^{(2)}=c_{i_{1}, i_{2}} c_{i_{3}, i_{4}}+c_{i_{1}, i_{4}} c_{i_{2}, i_{3}}-c_{i_{1}, i_{3}} c_{i_{2}, i_{4}}
$$

and more generally, for no pair to meet among $2 n$ walkers, one finds

$$
c_{i_{1}, i_{2} \ldots \ldots, i_{2 n-1}, i_{2 n}}^{(n)}=\frac{1}{2^{n} \cdot n !} \sum_{\sigma} \varepsilon(\sigma) c_{i_{\sigma(1), i_{\sigma(2)}}} \cdots c_{\left.i_{\sigma 12 n}-1\right), i_{\sigma(2 n)}}
$$


where the sum runs over all the permutations $\sigma$ of the indices $\left\{i_{1}, i_{2}, \ldots, i_{2 n}\right\}$, $\varepsilon(\sigma)$ is the signature of the permutation $\sigma$, and the matrix $c_{i, j}$ is antisymmetrized by defining, for $\tau_{i}>\tau_{j}$,

$$
c_{i, j}=-c_{j, i}<0 \quad \text { when } \quad i>j
$$

[note that (11) and (12) are Pfaffians, ${ }^{(21)}$ simply meaning that the sums in (11), (12) are over all possible pairings of the indices]. A proof of (11), (12) is given in Appendix A.

As explained in Appendix A, from the $c^{(n)}$, one can calculate the probabilities of all the coalescing schemes between $N$ walkers, in particular, the probability that $N$ walkers starting at times $t-\tau_{N}<t-\tau_{N-1}<\cdots$ $t-\tau_{1}$ become exactly $m$ walkers at time $t$. Moreover as in (11), (12) all the $c^{(\prime \prime)}$ can be expressed in terms of the matrix $c_{i, j}$, it is sufficient to know this matrix $c$.

One can calculate the matrix $c_{i, j}$ by the method of images. Consider first a single walker on the semiinfinite chain (1), (9) starting at the origin at $t=0$. The probability $p(x, t)$ of finding this walker on site $x$ at time $t$ evolves according to

$$
\frac{d p(x, t)}{d t}=p(x+1, t)+p(x-1, t)-2 p(x, t) \quad \text { for } \quad x>0
$$

and

$$
\frac{d p(0, t)}{d t}=p(1, t)-p(0, t)
$$

The solution for the initial condition $p(x, 0)=\delta_{x .0}$ is

$$
p(x, t)=\frac{1}{2 \pi} \int_{0}^{2 \pi} d \theta\{\cos (x \theta)+\cos [(x+1) \theta]\} \exp [-2(1-\cos \theta) t]
$$

which becomes in the long-time limit,

$$
p(x, t) \simeq \frac{1}{\sqrt{\pi t}} \exp -\frac{x^{2}}{4 t}
$$

Consider now two walkers starting at the origin at times $t-\tau_{j}<t-\tau_{i}$. The probability $c_{i, j}$ that they do not meet up to time $t$ is given by

$$
c_{i, j}=\sum_{0 \leqslant x \leqslant y} p\left(x, \tau_{i}\right) p\left(y, \tau_{j}\right)-p\left(x, \tau_{j}\right) p\left(y, \tau_{i}\right)
$$


If one uses the long-time expression of $p(x, t)$, one obtains for large $\tau_{i}$ and $\tau_{j}$

$$
c_{i, j} \simeq \frac{4}{\pi} \tan ^{-1}\left(\frac{\tau_{j}}{\tau_{i}}\right)^{1 / 2}-1
$$

\section{THE SEMIINFINITE CHAIN AT $\boldsymbol{N}$ DIFFERENT TIMES $T_{1}, T_{2}, \ldots, T_{N}$}

In Appendix A we calculate for the case $N=4$ the probabilities $P(m, t)$ in terms of the $4 \times 4$ matrix $c_{i, j}$. This gives for the probability $A_{4}\left(\tau_{1}, \tau_{2}, \tau_{3}, \tau_{4}\right)$ that $S_{0}\left(\tau_{1}\right)=S_{0}\left(\tau_{2}\right)=S_{0}\left(\tau_{3}\right)=S_{0}\left(\tau_{4}\right)$

$$
\begin{aligned}
A_{4}\left(\tau_{1}, \ldots, \tau_{4}\right)= & 1-\left(c_{1.2}+c_{2.3}+c_{3.4}\right) \frac{q-1}{q^{2}} \\
& -c_{1.4} \frac{(q-1)^{2}}{q^{2}}+c_{1.2 .3 .4}^{(2)} \frac{(q-1)^{2}}{q^{3}}
\end{aligned}
$$

The key to our solution is that we could generalize this expression to an arbitrary number $N$ of walkers. Let us define $\lambda$ and $\mu$ by

$$
\begin{aligned}
& \lambda=q-1 \\
& \mu=\frac{q-1}{q^{2}}
\end{aligned}
$$

We shall prove that the probability $A_{N}\left(\tau_{1}, \tau_{2}, \ldots, \tau_{N}\right)$ that $S_{0}\left(\tau_{1}\right)=$ $S_{0}\left(\tau_{2}\right)=\cdots S_{0}\left(\tau_{N}\right)$ is given by

$$
\begin{aligned}
& A_{N}\left(\tau_{1}, \tau_{2}, \ldots, \tau_{N}\right) \\
&=1-\mu \sum_{i=1}^{N-1} c_{i, i+1}+\mu^{2} \sum_{i<j} c_{i, i+1, j, j+1}^{(2)} \\
&-\mu^{3} \sum_{i<j<k} c_{i, i+1, j, j+1, k, k+1}^{(3)}+\cdots \\
&-\lambda\left\{\mu c_{1, N}-\mu^{2} \sum_{i} c_{1, i, i+1, N}^{(2)}+\mu^{3} \sum_{i<j} c_{1, i, i+1, j, j+1, N}^{(3)}-\cdots\right\}
\end{aligned}
$$

(note that as long as $N$ is finite, $A_{N}$ is the sum of a finite number of terms and is therefore a polynomial in the variable $\mu$ ).

To prove that $(18)$ is equivalent to $(8)$, we need to show that the weight of the events where the $N$ walkers end up being $m$ aggregates at 
time $t$ is $q^{1-m}$ in (8). Consider a situation where all the walkers starting at times $t-\tau_{N}<t-\tau_{N-1}<\cdots t-\tau_{1}$ end up forming $m$ clusters at time $t$. The weight $a_{m}$ of such an event in (18) is

$$
\begin{aligned}
a_{m}= & 1-(m-1) \mu+\frac{(m-2)(m-3)}{2 !} \mu^{2}-\frac{(m-3)(m-4)(m-5)}{3 !} \mu^{3} \cdots \\
& -\lambda\left(\mu-(m-3) \mu^{2}+\frac{(m-4)(m-5)}{2 !} \mu^{3} \cdots\right)
\end{aligned}
$$

(The case $m=1$ is special and $a_{1}=1$ ). This formula can be understood by looking at (18) term by term: if there are $m$ walkers left at time $t$, then there are $m-1$ pairs $i, i+1$ which do not meet, $(m-2)(m-3) / 2$ quadruples $i, i+1, j, j+1$ which do not meet, and so on. $a_{m}$ is a polynomial in $\mu$ which can be rewritten in a closed form

$$
\begin{aligned}
a_{m}= & \frac{1}{\sqrt{1-4 \mu}}\left[\left(\frac{1+\sqrt{1-4 \mu}}{2}\right)^{m+1}-\left(\frac{1-\sqrt{1-4 \mu}}{2}\right)^{m+1}\right] \\
& -\lambda \mu \frac{1}{\sqrt{1-4 \mu}}\left[\left(\frac{1+\sqrt{1-4 \mu}}{2}\right)^{m-1}-\left(\frac{1-\sqrt{1-4 \mu}}{2}\right)^{m-1}\right]
\end{aligned}
$$

Using (16) and (17), this gives $a_{m}=1 / q^{m-1}$ and therefore completes the proof that $(18)$ is the same as $(8)$.

There are several ways of rewriting (18). One can show (Appendix B) that (18) can be written as the square root of a determinant,

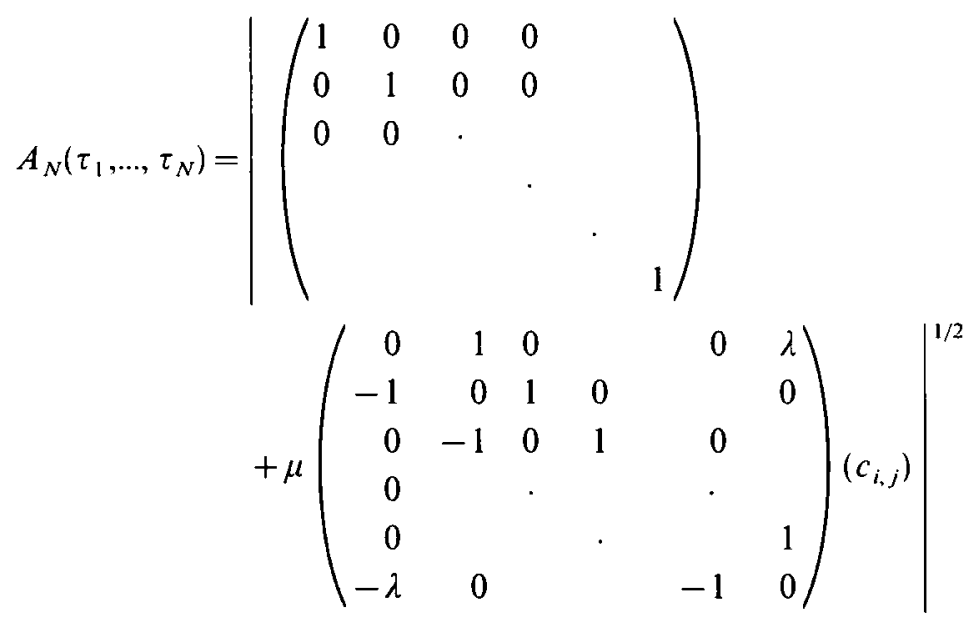

where $\left(c_{i j}\right)$ is the $N \times N$ matrix the elements of which are $c_{i j}$. Another way 
of rewriting (21) which will be more convenient in Section 4 to take the continuous limit is

$$
\begin{aligned}
A_{N}\left(\tau_{1}, \ldots, \tau_{N}\right)= & (\operatorname{det} M)^{1 / 2}\left\{1+\mu\left(c M^{-1}\right)_{2,1}-\mu\left(c M^{-1}\right)_{N-1 . N}\right. \\
& +\lambda \mu\left(c M^{-1}\right)_{N, 1}-\lambda \mu\left(c M^{-1}\right)_{1 . N} \\
& +\mu^{2}\left[\lambda\left(c M^{-1}\right)_{1.1}+\left(c M^{-1}\right)_{N-1.1}\right] \\
& \times\left[\lambda\left(c M^{-1}\right)_{N . N}+\left(c M^{-1}\right)_{2, N}\right] \\
& -\mu^{2}\left[\lambda\left(c M^{-1}\right)_{N .1}+\left(c M^{-1}\right)_{2.1}\right] \\
& \left.\times\left[\lambda\left(c M^{-1}\right)_{1 . N}+\left(c M^{-1}\right)_{N-1 . N}\right]\right\}^{1 / 2}
\end{aligned}
$$

where the matrix $M$ is given by

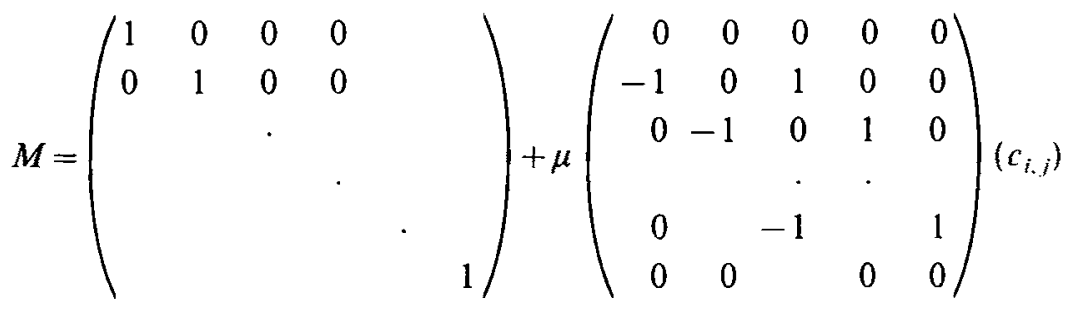

This is just a consequence of the fact that for any matrix $Q$ and vectors $\left|V_{1}\right\rangle,\left\langle W_{1}|,| V_{2}\right\rangle,\left\langle W_{2}\right|$

$$
\begin{aligned}
\operatorname{det}[Q & \left.+\left|V_{1}\right\rangle\left\langle W_{1}|+| V_{2}\right\rangle\left\langle W_{2}\right|\right] \\
= & {\left[\left(1+\left\langle W_{1}\left|M^{-1}\right| V_{1}\right\rangle\right)\left(1+\left\langle W_{2}\left|M^{-1}\right| V_{2}\right\rangle\right)\right.} \\
& \left.-\left\langle W_{1}\left|M^{-1}\right| V_{2}\right\rangle\left\langle W_{2}\left|M^{-1}\right| V_{1}\right\rangle\right] \operatorname{det} Q
\end{aligned}
$$

Remark. There is still another way (see Appendix B) of writing $A_{N}\left(\tau_{1}, \ldots, \tau_{N}\right)$ in terms of the antisymmetric matrix $b$ defined by

$$
b_{i, j}=-b_{j, i}=1-c_{i, j} \quad \text { for } \quad i<j
$$

and $A_{N}\left(\tau_{1}, \ldots, \tau_{N}\right)$ becomes

$$
\begin{aligned}
A_{N}\left(\tau_{1}, \ldots, \tau_{N}\right)= & \frac{1}{q^{N-1}}\left\{1+\sum_{i<j}(q-1)^{j-i} b_{i, j}\right. \\
& +\mu^{2} \sum_{i<j<k<1}(q-1)^{l-k+j-i} b_{i, j, k, I}^{(2)} \\
& \left.+\mu^{3} \sum_{i<j<k<1<m<n}(q-1)^{n-m+l-k+j-i} b_{i, j, k, I, m, n}^{(3)}+\cdots\right\}
\end{aligned}
$$


This expression has the same form (but with a different matrix $b$ ) as the one obtained in ref. 15 for the finite system [see (43) below]. As explained in Appendix B, this can be rewritten as

$$
A_{N}\left(\tau_{1}, \ldots, \tau_{N}\right)=\frac{1}{q^{N-1}} \sqrt{\operatorname{det}(I+\mu \Lambda b)}
$$

where the matrix $b$ is given in (23) and the antisymmetric matrix $A$ is defined by

$$
\Lambda_{i, j}=-\Lambda_{j, i}=-\lambda^{j-i} \quad \text { for } \quad i<j
$$

\section{EXACT EXPRESSION OF THE NUMBER OF PERSISTENT SPINS}

With the above expressions (18), (22) it is possible to take the limit $(N \rightarrow \infty)$ of a dense set of $\tau_{i}$ between two times $t_{1}$ and $t_{2}$. In this continuous limit the matrices $c_{i, j}$ and $M_{i, j}$ become kernels $c\left(t, t^{\prime}\right)$,

$$
c\left(t, t^{t}\right)=-c\left(t^{\prime}, t\right)
$$

with $c\left(t, t^{\prime}\right)>0$ if $t<t^{\prime}$, and $M\left(t, t^{\prime}\right)$

$$
M\left(t, t^{\prime}\right)=\delta\left(t-t^{\prime}\right)+2 \mu \frac{d}{d t} c\left(t, t^{\prime}\right)
$$

The matrix product as well as inversion or exponentiation have obvious generalizations in this continuum limit. For example,

$$
M^{2}\left(t, t^{\prime}\right)=\int_{t^{\prime}}^{t^{2}} d t^{\prime \prime} M\left(t, t^{\prime \prime}\right) M\left(t^{\prime \prime}, t^{\prime}\right)
$$

In this large- $N$ limit, starting from (18), one finds that the probability $R\left(q ; t_{1}, t_{2}\right)$ that the spin at the origin of an infinite chain does not flip during the time interval $\left(t_{1}, t_{2}\right)$ is

$$
\begin{aligned}
R\left(q ; t_{1}, t_{2}\right)= & 1+\mu \int_{t_{1}}^{t_{2}} \frac{d}{d t} c(u, u) d u+\frac{\mu^{2}}{2}\left\{\int_{t_{1}}^{t_{2}} \frac{d}{d t} c(u, u) d u\right\}^{2} \\
& +\mu^{2} \int_{t_{1}}^{t_{2}} d u \int_{u}^{t_{2}}\left[\frac{d}{d t} c(u, v) \frac{d}{d t^{\prime}} c(u, v)-c(u, v) \frac{d^{2}}{d t d t^{\prime}} c(u, v)\right] d v
\end{aligned}
$$




$$
\begin{aligned}
& -\lambda\left\{\mu c\left(t_{1}, t_{2}\right)+\mu^{2} c\left(t_{1}, t_{2}\right) \int_{t_{1}}^{t_{2}} \frac{d}{d t} c(u, u) d u\right. \\
& \left.+\mu^{2} \int_{t_{1}}^{t_{2}}\left[c\left(t_{1}, u\right) \frac{d}{d t^{\prime}} c\left(u, t_{2}\right)-c\left(u, t_{2}\right) \frac{d}{d t^{\prime}} c\left(t_{1}, u\right)\right] d u\right\}+O\left(\mu^{3}\right)
\end{aligned}
$$

This is an expansion in powers of $\mu$, and therefore, according to (17), is valid for $q$ close to 1 or $\infty$.

One can also take the $N \rightarrow \infty$ limit in (22). This leads to an expression valid for arbitrary $t_{1}, t_{2}$, and $\mu$ :

$$
\begin{aligned}
R\left(q ; t_{1}, t_{2}\right)= & \left\{1+\mu \tilde{c}\left(t_{1}, t_{1}\right)-\mu \tilde{c}\left(t_{2}, t_{2}\right)+\lambda \mu \tilde{c}\left(t_{2}, t_{1}\right)-\lambda \mu \tilde{c}\left(t_{1}, t_{2}\right)\right. \\
& \left.+\mu^{2}\left(1-\lambda^{2}\right) \tilde{c}\left(t_{1}, t_{2}\right) \tilde{c}\left(t_{2}, t_{1}\right)-\mu^{2}\left(1-\lambda^{2}\right) \tilde{c}\left(t_{1}, t_{1}\right) \tilde{c}\left(t_{2}, t_{2}\right)\right\}^{1 / 2} \\
& \times \exp \left(\frac{1}{2} \operatorname{tr} \log M\right)
\end{aligned}
$$

where

$$
\tilde{c}=c M^{-1}
$$

This expression can be further simplified by using (C1)-(C3) of Appendix $\mathrm{C}$ relating the four matrix elements $\tilde{c}\left(t_{1}, t_{1}\right), \tilde{c}\left(t_{2}, t_{2}\right), \tilde{c}\left(t_{2}, t_{1}\right)$, and $\tilde{c}\left(t_{1}, t_{2}\right)$. One then gets

$$
R\left(q ; t_{1}, t_{2}\right)=\left[\sqrt{1-\mu \tilde{c}\left(t_{2}, t_{2}\right)}-\lambda \sqrt{-\mu \tilde{c}\left(t_{2}, t_{2}\right)}\right] \exp \left(\frac{1}{2} \operatorname{tr} \log M\right)
$$

Once $R\left(q ; t_{1}, t_{2}\right)$ is known, $r(q, t)$ for an infinite chain follows through (10). So we have an exact expression of $r(q, t)$ in terms of the matrix $c\left(t, t^{\prime}\right)$, which is known:

$$
\begin{aligned}
c\left(t, t^{\prime}\right) & =\sum_{0 \leqslant x \leqslant y} p(x, t) p\left(y, t^{\prime}\right)-p\left(x, t^{\prime}\right) p(y, t) \\
\operatorname{tr} \log M & =-\sum_{n=1}^{\infty} \frac{(-2 \mu)^{\prime \prime}}{n} \int_{t^{\prime}}^{t_{2}} d \tau_{1} \ldots \int_{t_{1}}^{t_{2}} d \tau_{n} \frac{d}{d t} c\left(\tau_{1}, \tau_{2}\right) \ldots \frac{d}{d t} c\left(\tau_{n}, \tau_{1}\right)
\end{aligned}
$$

and

$$
\begin{aligned}
\tilde{c}\left(t_{2}, t_{2}\right)= & \sum_{n=1}^{\infty}(-2 \mu)^{n} \int_{t_{1}}^{t_{2}} d \tau_{1} \cdots \int_{t_{1}}^{t_{1}} d \tau_{n} c\left(t_{2}, \tau_{1}\right) \\
& \times \frac{d}{d t} c\left(\tau_{1}, \tau_{2}\right) \cdots \frac{d}{d t} c\left(\tau_{n}, t_{2}\right)
\end{aligned}
$$




\section{THE ASYMPTOTICS}

When $t_{1}$ and $t_{2}$ are both large, one can replace $c\left(t, \tau^{\prime}\right)$ in (30) and (31) by its asymptotic expansion. We have seen from (15)

$$
c\left(\tau, \tau^{\prime}\right) \simeq g\left(\frac{\tau}{\tau^{\prime}}\right)
$$

where

$$
g(z)=\frac{4}{\pi} \tan ^{-1}\left(\frac{1}{z}\right)^{1 / 2}-1=1-\frac{4}{\pi} \tan ^{-1} \sqrt{z}
$$

For $t_{2} \gg t_{1} \gg 1$, the leading behavior of $(30)$ is easy to obtain,

$$
\begin{aligned}
& \int_{t_{1}}^{\prime_{2}} d \tau_{1} \cdots \int_{t_{1}}^{t_{2}} d \tau_{n} \frac{d}{d t} c\left(\tau_{1}, \tau_{2}\right) \cdots \frac{d}{d t} c\left(\tau_{n}, \tau_{1}\right) \\
& \quad \simeq \int_{\log t_{1}}^{\log t_{2}} d u_{1} \cdots \int_{\log t_{1}}^{\log t_{2}} d u_{n} g^{\prime}\left(e^{u_{1}-u_{2}}\right) \cdots g^{\prime}\left(e^{u_{n}-u_{1}}\right)
\end{aligned}
$$

This has a Toeplitz form and standard methods can be used ${ }^{(22-24)}$ to evaluate its leading behavior: as $g^{\prime}\left(e^{u-v}\right) \rightarrow 0$ when $|u-v| \rightarrow \infty$, the previous integral is dominated by situations where all the $u_{i}$ are grouped around $u_{1}$. Therefore, for large $t_{2} / t_{1}$,

$$
\begin{aligned}
\int_{\log t_{1}}^{\log t_{2}} d u_{1} & \cdots \int_{\log t_{1}}^{\log t_{2}} d u_{n} g^{\prime}\left(e^{u_{1}-u_{2}}\right) \cdots g^{\prime}\left(e^{\left.i_{n}-t_{1}\right)}\right) \\
\simeq & \log \left(\frac{t_{2}}{t_{1}}\right) \int_{-\infty}^{\infty} d v_{1} \cdots \int_{-\infty}^{\infty} d v_{n} \\
& \times g^{\prime}\left(e^{v_{1}}\right) g^{\prime}\left(e^{i_{2}}\right) \cdots g^{\prime}\left(e^{i_{n}}\right) e^{i_{1}+t_{2}+\cdots v_{n}} \delta\left(v_{1}+v_{2}+\cdots v_{n}\right) \\
= & \log \left(\frac{t_{2}}{t_{1}}\right) \frac{1}{2 \pi} \int_{-\infty}^{\infty} d k\left[\int_{-\infty}^{\infty} e^{i k v^{v}} e^{v} g^{\prime}\left(e^{\prime \prime}\right) d v\right]^{\prime \prime}
\end{aligned}
$$

and this gives the asymptotic behavior of $(30)$,

$$
\operatorname{tr} \log M \simeq \log \left(\frac{t_{2}}{t_{1}}\right) \frac{1}{2 \pi} \int_{-\infty}^{\infty} d k \log \left[1+2 \mu \int_{-\infty}^{\infty} e^{i k r} e^{\prime \prime} g^{\prime}\left(e^{\prime \prime}\right) d v\right]
$$

[note that $e^{i} g^{\prime}\left(e^{i}\right)$ is even, so that (34) is real]. 
In Appendix $\mathrm{C}$, we calculate the matrix element $\tilde{c}\left(t_{2}, t_{2}\right)=\left(c M^{-1}\right)_{t_{2} \cdot t_{2}}$ in the limit $t_{2}$ large, $(\mathrm{C} 5)$. In that limit this gives

$$
\begin{aligned}
& {\left[1-\mu \tilde{c}\left(t_{2}, t_{2}\right)\right]^{1 / 2}-\lambda\left[-\mu \tilde{c}\left(t_{2}, t_{2}\right)\right]^{1 / 2}} \\
& \quad \simeq\left(\frac{1}{2}+\frac{1-2 \mu}{2 \sqrt{1-4 \mu}}\right)^{1 / 2}-\lambda\left(-\frac{1}{2}+\frac{1-2 \mu}{2 \sqrt{1-4 \mu}}\right)^{1 / 2}
\end{aligned}
$$

and using from (16) and (17) that $\sqrt{1-4 \mu}=|q-2| / q$, one obtains

$$
\begin{aligned}
& {\left[1-\mu \tilde{c}\left(t_{2}, t_{2}\right)\right]^{1 / 2}-\lambda\left[-\mu \tilde{c}\left(t_{2}, t_{2}\right)\right]^{1 / 2}} \\
& \quad \simeq\left(\frac{q^{2}-2 q+2+q|q-2|}{2 q|q-2|}\right)^{1 / 2}-(q-1)\left(\frac{q^{2}-2 q+2-q|q-2|}{2 q|q-2|}\right)^{1 / 2}
\end{aligned}
$$

One then has to distinguish two cases:

1. For $q<2$, the prefactor $(35)$ has a limit $\sqrt{q(2-q)}$ when $t_{2} \rightarrow \infty$ and so (29) leads to

$$
R\left(q ; t_{1}, t_{2}\right) \sim\left(\frac{t_{1}}{t_{2}}\right)^{g(q) / 2}
$$

where

$$
\theta(q)=-\frac{1}{2 \pi} \int_{-\infty}^{\infty} d k \log \left[1+2 \mu \int_{-\infty}^{\infty} e^{i k k^{\prime}} e^{i} g^{\prime}\left(e^{v}\right) d v\right]
$$

When the explicit expression (33) of $g(z)$ is used, one recovers (7). In deriving (7) from (36), it is useful to remember that

$$
2 \int_{-\infty}^{\infty} d u \frac{e^{2 i k u+t}}{1+e^{2 t t}}=\frac{\pi}{\cosh \pi k}
$$

and

$$
\int_{-\infty}^{\infty} d y \log \left(1-\frac{a}{\cosh y}\right)=\frac{\pi^{2}}{4}-4\left[\cos ^{-1}\left(\frac{1-a}{2}\right)^{1 / 2}\right]^{2}
$$

2. For $q>2$, as (30) depends only on $\mu=(q-1) / q^{2}$, one could believe that the expression (36) of $\theta(q)$ remains the same under the transformation $q \rightarrow q /(q-1)$, which leaves $\mu$ unchanged. However, for $q>2$, the prefactor (29), (35) vanishes as a power law when $t_{2} \rightarrow \infty$ and this gives an additional contribution to $\theta(q)$.

$$
\theta(q)=2 k_{0}(\mu)-\frac{1}{2 \pi} \int_{-\infty}^{\infty} d k \log \left[1+2 \mu \int_{-\infty}^{\infty} e^{i k n} e^{u} g^{\prime}\left(e^{v}\right) d v\right]
$$


where $k_{0}(\mu)$ is the smallest positive solution

$$
1+2 \mu \int_{-\infty}^{\infty} e^{k_{v} \prime} e^{r} g^{\prime}\left(e^{r}\right) d v=0
$$

Before arguing why this is so, let us note that this new expression (37) of $\theta(q)$ leads also (for $q>2$ ) to (7) when $g(z)$ is given by (33). In fact, it is an exercise in complex analysis to show that $(37)$ is the analytic continuation of (36) to the range $q>2$ : as $4 \mu \rightarrow 1$, two zeros $i k_{0}$ and $-i k_{0}$ of the argument of the logarithm in (36) approach the real axis in the plane of the complex variable $k$ and the analytic continuation (37) of (36) corresponds to a deformation of the path of integration over $k$ to go around these two zeros.

The fact that the prefactor (29), (35) decays like $t_{2}^{-k_{0}}$ can be understood as follows. Let $\delta$ denote the difference between the matrix element $\left(c M^{-1}\right)_{t_{2}, t_{2}}$ and its limiting value $(\mathrm{C} 5)$,

$$
\delta=\left(c M^{-1}\right)_{t_{2, t_{2}}}-\frac{1}{2 \mu}-\frac{1-2 \mu}{2 \mu \sqrt{1-4 \mu}}
$$

Then the prefactor of $R\left(q ; t_{1}, t_{2}\right)$ in (29) can be expanded in powers of $\delta$ and the first term in the expansion is, for $q>2$,

$$
\sqrt{1-\mu \tilde{c}\left(t_{2}, t_{2}\right)}-\lambda \sqrt{-\mu \tilde{c}\left(t_{2}, t_{2}\right)}=\frac{(q-2)^{3 / 2}}{2 q^{1 / 2}} \delta+O\left(\delta^{2}\right)
$$

Thus we need only evaluate the asymptotic expression of $\delta$. For this it is convenient to consider the following matrix element:

$$
\varepsilon(u)=\frac{1}{2 \mu}+\left(1-\frac{1}{2 \mu}\right)\left(c M^{-1}\right)_{t_{1} . u}-\left(c M^{-1}\right)_{t_{2} . u}
$$

From (C4), (C5), when $t_{2}$ is large, $\varepsilon\left(t_{2}\right)$ goes to zero and the first term in its expansion (C3) in powers of $\delta$ is

$$
\varepsilon\left(t_{2}\right)=\left[\frac{q(q-2)}{2(q-1)}\right]^{2} \delta+O\left(\delta^{2}\right)
$$

The asymptotic expression of $\delta$ can be understood from the integral equation satisfied by $\varepsilon(u)$

$$
\varepsilon(u)=\frac{1}{2 \mu}\left[1-c\left(t_{1}, u\right)\right]-2 \mu \int_{t_{1}}^{t_{2}} d \tau \varepsilon(\tau) \frac{d}{d \tau} c(\tau, u)
$$


Let us consider this equation in the following three regions: (a) $u \sim t_{1}$, (b) $t_{1} \ll u \ll t_{2}$, (c) $u \sim t_{2}$.

In region (b), if we neglect the inhomogeneous term,

$$
\varepsilon(u)=-2 \mu \int_{t_{1}}^{\prime 2} d \tau \varepsilon(\tau) \frac{d}{d \tau} c(\tau, u)
$$

and if we use the fact that $(d / d \tau) c(\tau, u)$ is nonzero only when $\tau / u$ is of order 1 , we find that $\varepsilon(u)$ can only decay as $u^{-k_{11}}$, where $k_{0}$ is a solution of (38). In fact, (38) has in general several solutions and the most general decaying $\varepsilon(u)$ solution of the homogeneous equation is a priori a sum of power laws, but as we are interested in the leading behavior of $\varepsilon(u)$ for large $u$, we only need to consider the slowest decay given by the smallest positive solution of (38). One can check that in region (b), $u^{-k_{0}} \gg(1 / 2 \mu)$ $\left[1-c\left(t_{1}, u\right)\right]$, so that the inhomogeneous term is really negligible.

This power-law solution in region (b) joins the solutions in regions (a) and (c), where finding $\varepsilon(u)$ becomes a Wiener-Hopf problem in the variable $\log u$. We solved this Wiener-Hopf problem for several simple choices of $(d / d \tau) c(\tau, u)$ and we found the expected matching with the solution of the homogeneous equation in region (b). This convinced us that

$$
\delta \sim \varepsilon\left(t_{2}\right) \sim t_{2}^{-k_{0}}
$$

where $k_{0}$ is the smallest positive solution of (38).

Remark. We have shown so far that for $1 \ll t_{1} \ll t_{2}$,

$$
R\left(q ; t_{1}, t_{2}\right) \sim t_{2}^{-\theta(q)}
$$

What we need in (10) is the decay for large $t_{2}$ of $R\left(q ; 0, t_{2}\right)$. It turns out that the condition that $t_{1}$ is large can be relaxed, without changing the exponent. This is a consequence of the inequalities

$$
\frac{1}{q} R\left(q ; 0, t_{1}\right) R\left(q ; t_{1}, t_{2}\right) \leqslant R\left(q ; 0, t_{2}\right) \leqslant R\left(q ; t_{1}, t_{2}\right)
$$

The right inequality is trivial, whereas the left one can be proved by representing each term by coalescing random walkers as in Section 2 and by noticing. that more coalescences take place in $R\left(q ; 0, t_{2}\right)$ than in the product $R\left(q ; 0, t_{1}\right) R\left(q ; t_{1}, t_{2}\right)$.

Remark. The difference between the cases $q<2$ and $q>2$ is a direct consequence of the splitting in (18) and (29) between the term proportional to $\lambda$ and the term independent of $\lambda$. In $(20), 1 / q^{m-1}=a_{m}$ was rewritten as a sum of two terms, 


$$
\begin{aligned}
\frac{1}{q^{m-1}}= & \frac{q}{2-q}\left[\frac{1}{q^{m+1}}-\left(\frac{q-1}{q}\right)^{m+1}\right] \\
& -\lambda \frac{q-1}{q(2-q)}\left[\frac{1}{q^{m-1}}-\left(\frac{q-1}{q}\right)^{m-1}\right]
\end{aligned}
$$

From (8), it follows that $R\left(q ; t_{1}, t_{2}\right)$ is evaluated as a sum of two terms

$$
\begin{aligned}
R\left(q ; t_{1}, t_{2}\right)= & \frac{q}{2-q}\left(\frac{1}{q^{2}} R\left(q ; t_{1}, t_{2}\right)-\left(\frac{q-1}{q}\right)^{2} R\left(\frac{q}{q-1} ; t_{1}, t_{2}\right)\right) \\
& -\lambda \frac{q-1}{q(2-q)}\left(R\left(q ; t_{1}, t_{2}\right)-R\left(\frac{q}{q-1} ; t_{1}, t_{2}\right)\right)
\end{aligned}
$$

When the contribution of the second ( $\lambda$-dependent) term is not taken into account (as when computing $\exp [\operatorname{tr} \log M / 2]$ after extraction of the $\lambda$-dependent $2 \times 2$ matrix $)$ the leading asymptotic behavior of $R\left(q ; t_{1}, t_{2}\right)$ is estimated as a sum of those of $R\left(q ; t_{1}, t_{2}\right)$ and $R\left(q /(q-1) ; t_{1}, t_{2}\right)$. For $0<q<2, R\left(q /(q-1) ; t_{1}, t_{2}\right) \ll R\left(q ; t_{1}, t_{2}\right)$, so that the $\lambda$-independent term decays like $R\left(q ; t_{1}, t_{2}\right)$. On the contrary, for $q>2$, the $\lambda$-independent sum behaves asymptotically like $R\left(q /(q-1) ; t_{1}, t_{2}\right) \gg R\left(q ; t_{1}, t_{2}\right)$. This spurious leading behavior is exactly canceled in $(40)$ by the $\lambda$-dependent term and the exponent $\theta(q)$ recovered only when the contribution of the $\lambda$-dependent sum is taken into account.

Remark. When $t_{1}$ and $t_{2}$ are both large, $R\left(q ; t_{1}, t_{2}\right)$ become a scaling function of the ratio $t_{1} / t_{2}$. This scaling function given by (29) is universal, in the sense that it would remain the same if short-range correlations were present in the initial condition. In the limit $1 \ll t_{1} \ll t_{2}$, $R\left(t_{1}, t_{2} ; q\right) \sim A\left(t_{1} / t_{2}\right)^{(t q) / 2}$, and one could compute the prefactor $A$ using the theory of Toeplitz determinants. ${ }^{(22-24)}$

\section{FINITE SYSTEMS AND FINITE-SIZE SCALING}

When one considers the spin problem (1) for a finite system of size $L$, there is a single domain left in the system after a time $t \gg L^{2}$, since the size of the domains grows like $\sqrt{t}$. The dynamics then stops. Each spin therefore has a finite probability $\rho_{L}(q)$ to never flip (between $t=0$ and $t=\infty$ ). One expects $\rho_{L}(q)$ to be comparable to the fraction of spins in the infinite system which never flip between $t=0$ and $t \sim L^{2}$, so that

$$
\rho_{L}(q) \sim r\left(q, L^{2}\right) \sim L^{-2(q(q)}
$$

$\theta(q)$ can therefore be determined from the asymptotics of $\rho_{L}(q)$ for large $L$. 
In an earlier letter ${ }^{(15)}$ we obtained the exact expression of $\rho_{L}(q)$ by analyzing the steady state of the $(A+A \rightarrow A)$ coalescing random walk model on a finite ring with periodic boundary conditions and by using finite-size scaling. We recall here this alternative approach. In Section 7, we will show that the same expression of $\rho_{L}(q)$ can be recovered by a freefermion theory.

The reaction-diffusion model is defined on a ring of $L$ sites as follows. The origin ( site 0 , which is identical to site $L$ ) is always occupied and is a source of particles. All other sites can be either occupied or empty. As on the infinite line, during every infinitesimal time interval $\Delta t$, each particle hops with probability $\Delta t$ to its right neighbor and with probability $\Delta t$ to its left neighbor (and does not move with probability $1-2 \Delta t$ ); if two particles occupy the same site, they instantaneously coagulate $(A+A \rightarrow A)$; in addition, whenever the particle at the origin jumps to one of its neighbors, a new particle is instantaneously produced at the origin.

The connection ${ }^{(14.15)}$ between the spin problem and the reaction-diffusion model was recalled in Section 2 . The probability $p_{L}(q, t)$ that the spin at the origin did not flip between time 0 and $t$ starting from a random initial condition is related to the probability of finding $m$ particles at time $t$ in the reaction-diffusion model (starting from the configuration where only the origin is occupied) through (8),

$$
p_{L}(q, t)=\sum_{m=1}^{L} P(m, t) \frac{1}{q^{m-1}}
$$

Therefore the probability $p_{L}(q)$ that the spin at the origin never flips (from $t=0$ to $\infty)$ is related to the probabilities $P_{\infty}(m)$ of finding $m$ particles in the steady state of the reaction-diffusion model

$$
\rho_{L}(q)=\sum_{m=1}^{L} P_{\infty}(m) \frac{1}{q^{m-1}}
$$

In ref. 15 we obtained the following exact expression for the probability $p_{L}(q)$ :

$$
\begin{aligned}
\rho_{L}(q)= & \frac{1}{q^{L-1}}\left[1+\sum_{1 \leqslant i<j \leqslant L}(q-1)^{j-i} B_{i j}\right. \\
& \left.+\sum_{1 \leqslant i<j<k<1 \leqslant L}(q-1)^{j-i+1-k} B_{i, j, k, l}^{(2)}+\cdots\right]
\end{aligned}
$$


where the antisymmetric matrix $B_{i, j}$ is a solution for $1 \leqslant i<j \leqslant L$ of

$$
B_{i+1, j}+B_{i-1, j}+B_{i, j+1}+B_{i, j-1}-4 B_{i, j}=-2 \delta_{i+1, j}
$$

The boundary conditions needed to obtain the solution of these equations can be taken into account by the convertions $B_{0, i}=B_{i, L+1}=0$ (note that in ref. 15 we had the convention $B_{i, i}=1$, whereas here we choose $B_{i, i}=0$; as this matrix element appears nowhere, its value has no effect on the results). As an illustrative example, for $L=4$, the solution of (44) is $B_{1,2}=$ $B_{3,4}=26 / 44 ; \quad B_{1,3}=B_{2,4}=16 / 44 ; \quad B_{1,4}=8 / 44 ; \quad B_{2,3}=30 / 44$. Here $B^{(2)}$, $B^{(3)}, \ldots, B^{(n)}, \ldots$ are Pfaffians of the matrix $B$ defined as above (11), (12). As shown in (B8), (B9) of Appendix B, the sum in (43) can be rewritten as a determinant and one has the alternative expression

$$
\rho_{L}(q)=\frac{1}{q^{L-1}} \sqrt{\operatorname{det}(I+A B)}
$$

where the antisymmetric matrix $\Lambda$ is given in (26).

A derivation of (43) was given in ref. 15. It is based on the fact that $B_{i, j}$ for $1 \leqslant i<j \leqslant L$ is the steady-state probability that the segment $\{i, i+1, \ldots, j-i\}$ contains no particle. The $B_{i, j}$ obey closed kinetic equations ${ }^{(25-28)}$ Generalized such quantities were defined by introducing the probabilities $B_{i_{1}, i_{2} \ldots, i_{2 n-1}, i_{2 n}}^{(n)}$ that there is no particle in any of the disconnected segments $\left\{i_{1}, i_{1}+1, \ldots, i_{2}-1\right\},\left\{i_{3}, \ldots, i_{4}-1\right\}, \ldots,\left\{i_{2 n-1}, \ldots, i_{2 n}-1\right\}$ with $i_{1}<i_{2}<\ldots<i_{2 n}$. An examination of the kinetic equations that these quantities obey then showed that they can be expressed in terms of the $B_{i, j}$ and are in fact given by the Pfaffians of the matrix $B$.

Having obtained the exact expression (45), it remains to extract $\theta(q)$ from its large- $L$ behavior (41). We could not calculate the determinant in (45) for general $q$. However, working in Fourier space, we found the following expression valid for $q=0$ (besides the trivial case $q=1$ ):

$$
\sqrt{\operatorname{det}(I+\Lambda B)}
$$

$$
=2^{-L(L-1) / 2} \prod_{\substack{k \text { even } \\ 2 \leqslant k \leqslant L}} \prod_{\substack{k^{\prime} \text { odd } \\ 1 \leqslant k^{\prime} \leqslant L}}\left(\frac{2}{2-\cos [k \pi /(L+1)]-\cos \left[k^{\prime} \pi /(L+1)\right]}\right)
$$

(as this expression is used nowhere below, we do not give the derivation). For other values of $q$, we could only determine its asymptotic behavior for large $L$. As for the semiinfinite system, it is convenient to introduce instead 
of $B$ the antisymmetric matrix $C$ such that $C_{i, j}=1-B_{i, j}$ for $i<j$. As shown in Appendix B, (45) can be rewritten as

$$
\rho_{L}(q)=\sqrt{\operatorname{det}(I+a C)}
$$

where $a$ is the antisymmetric matrix (B2) with nonzero elements given by $a_{i, i+1}=-a_{i+1, i}=\mu, a_{1, L}=-a_{L, 1}=\lambda \mu$. One can note the similarity of (47) with (21) obtained for the semiinfinite system.

For large $L, C_{i, j}$ in (47) becomes a smooth and slowly varying function $C(x, y)$ of $x=i / L$ and $y=j / L$ except in the two small symmetric corners $\{0<x<\varepsilon, 0<y<\varepsilon\}$ and $\{0<1-x<\varepsilon, 0<1-y<\varepsilon\}$ with fixed $\varepsilon \ll 1$. There, the large- $L$ limit of $C(x, y)$ for fixed $x$ and $y$ is

$$
\begin{aligned}
C(x, y)=C(1-y, 1-x) & =f(x / y) \\
& =1-(4 / \pi) \tan ^{-1}(x / y) \quad \text { for } \quad 0<x, y<\varepsilon
\end{aligned}
$$

It precisely corresponds to the continuum limit expression (32), (33) of the semiinfinite system given that distances scale like square roots of time separations. We convinced ourselves, by looking at the singular behavior of the successive terms in the small- $\mu$ expansion of (47), that the behavior of $C(x, y)$ in these two small corners is responsible for the power-law decay of the determinant (47). Thus the large- $L$ behavior of (47) can be obtained along lines similar to those explained in Section 5. First, the contribution of the rank-two matrix can be explicitly extracted as in (22). This leads us to analyze $\sqrt{\operatorname{det}(I+\mu D)}$, where the matrix $D$ is defined by

$$
\begin{aligned}
& D_{i, j}=C_{i+1, j}-C_{i-1, j} \quad \text { for } \quad 2 \leqslant i \leqslant L-1 \\
& D_{1, j}=D_{L, j}=0
\end{aligned}
$$

The two corners both give contributions to the power-law decay which do not interfere (this can be checked in the small- $\mu$ expansion)

$$
\operatorname{det}(I+\mu D) \sim\left[\operatorname{det}\left(I+\mu D_{i j}\right)_{1 \ll i, j \ll \varepsilon L}\right]\left[\operatorname{det}\left(I+\mu D_{i j}\right)_{1 \ll L-j, L-i \ll \varepsilon L}\right]
$$

The two determinants on the r.h.s. of (50) are equal. They can be evaluated as in (30) by computing successive powers of the matrix $D$ in the continuum limit

$$
(I+\mu D)_{x, y}=\delta(x-y)+2 \mu \partial_{x} f(x / y)
$$


where $f$ is given by (48). This leads to

$$
\begin{aligned}
\theta(q) & =-\lim _{L \rightarrow \infty} \frac{\log \operatorname{det}(I+\mu D)}{2 \log L} \\
& =-\frac{1}{4 \pi} \int_{-\infty}^{\infty} d k \log \left[1+2 \frac{q-1}{q^{2}} \int_{-\infty}^{\infty} d x e^{i k x} e^{-x} f^{\prime}\left(e^{-x}\right)\right]
\end{aligned}
$$

which is identical to $(36)$, as $g(z)=f(\sqrt{z})$. As explained in Section 5, this expression is only valid for $1<q<2$ (when the contribution coming from the prefactor has a nonzero large- $L$ limit). As in Section 5 , for $q>2$, the prefactor gives an extra contribution [much harder to calculate here than in Section 5 because of the presence of the two corners and the fact that (48) is not valid everywhere in the matrix ] leading to $\theta(q)$ given by the analytic continuation of (52) outside the range $1<q<2$.

\section{THE FERMIONIC APPROACH}

In this section we show how (43) can be derived directly using fermions. Let us consider a $q$-state Potts model on a ring of $L$ sites evolving according to Glauber dynamics. We want to calculate the probability that the spin at position $0 \equiv L$ never flips when the initial condition is random. Each site in the initial condition is given one of the $q$ colors at random. Because the dynamics is like the dynamics of a voter model (1), one only needs to know which are the spins identical to the spin at the origin and which are the spins different in the initial condition.

\subsection{The Ising Basis}

Let us denote a configuration by a sequence of + signs if the spin at site $i$ is identical to the spin at the origin and - signs if it is different. So an initial condition can be represented by a vector with $L-1$ Ising spins $|(+)+--+-+\cdots-\rangle$ (the extra spin in parentheses being the spin at the origin, which is of course always + ). The weight of such a configuration is $(q-1)^{n-} / q^{L-1}$, where $n_{-}$is the number of - signs $[\mathrm{a}+$ corresponds to a spin identical to the spin at the origin and therefore has a weight $1 / q$ and a - is a spin with a color different from the spin at the origin and therefore has a weight $(q-1) / q]$. In other words the initial condition can be written as

$$
\left|P_{0}\right\rangle=\prod_{i=1}^{L-1}\left(\frac{1}{q}+\frac{q-1}{q} \sigma_{i}^{-}\right)|(+)+++\cdots+\rangle
$$


If $\left|P_{1}\right\rangle$ is the vector which has for components the probabilities of the $2^{L-1}$ configurations at time $t$, given that the spin at the origin has never flipped up to time $t$, the vector $\left|P_{1}\right\rangle$ evolves according to

$$
\frac{d}{d t}\left|P_{\imath}\right\rangle=H\left|P_{1}\right\rangle
$$

where

$$
H=g_{1}+h_{1,2}+\cdots+h_{L-2, L-1}+g_{L-1}
$$

with

$$
g_{1}=\sigma_{1}^{+}+\sigma_{1}^{z}-1 ; \quad g_{L-1}=\sigma_{L-1}^{+}+\sigma_{L-1}^{z}-1
$$

and

$$
\begin{aligned}
& h_{i, i+1}=-1+\sigma_{i}^{z} \sigma_{i+1}^{z}
\end{aligned}
$$

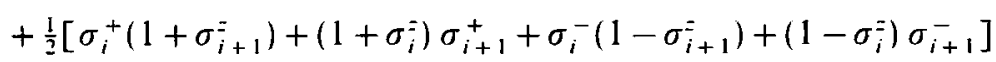

It is easy to check that $h_{i, i+1}$ expresses just the Glauber dynamics, whereas $g_{1}$ and $g_{L-1}$ cause all the events to vanish for which the spin at the origin flips at least once.

The main difference between this Hamiltonian and the usual spinchain Hamiltonians is that it does not conserve the total spin $\sum_{i=1}^{L-1} \sigma_{i}^{z}$.

Clearly

$$
\left|P_{1}\right\rangle=e^{H t}\left|P_{0}\right\rangle
$$

and $\rho_{L}(q)$ is just given by

$$
\rho_{L}(q)=\lim _{t \rightarrow \infty}\left\langle(+)+++\cdots+\left|\sum_{i=1}^{L-1}\left(1+\sigma_{i}^{+}\right) e^{H t}\right| P_{0}\right\rangle
$$

as the vector $\langle(+)+++\cdots+| \Pi\left(1+\sigma_{i}^{+}\right)$gives the sum of the weights of all the configurations.

Remark. The Hamiltonian $H$ [or in fact its transpose (D4)] can also be obtained from the reaction-diffusion model of Section 6, as explained in Appendix D. 


\subsection{The Basis of the Defects}

The effect of $H$ (zero-temperature Glauber dynamics) is that the number of defects +- or -+ never increases. This suggests that we define a set of spin variables $\mu_{i}, i=1, \ldots, L$, which live on the bonds of the previous lattice and index the position of these defects. That is, $\mu_{i}$ takes the value + if the values of the spins at sites $i-1$ and $i$ are opposite and - otherwise:

$$
\mu_{i}=\left\{\begin{array}{lll}
-1, & \text { if } & \sigma_{i-1}=\sigma_{i} \\
+1, & \text { if } & \sigma_{i-1} \neq \sigma_{i}
\end{array}\right.
$$

Periodic boundaries imply that the number of defects is always even. In this new basis the initial condition becomes

$$
\begin{aligned}
\left|P_{0}^{\prime}\right\rangle= & \left(\frac{1}{q}\right)^{L-1} \sum_{p} \sum_{x_{1}<y_{1}<x_{2}<y_{2} \cdots<y_{p}}\left(\prod_{j=1}^{p}(q-1)^{y_{j}-x_{j}}\right) \\
& \times \sigma_{x_{1}}^{+} \sigma_{y_{1}}^{+} \cdots \sigma_{x_{p}}^{+} \sigma_{y_{p}}^{+} \mid---\cdots->
\end{aligned}
$$

This expression is rather easy to understand: each time a pair of defects are created at positions $x_{j}$ and $y_{j}$, this corresponds to having all the spins in the previous basis becoming negative between these two consecutive defects and so the weight is multiplied by $(q-1)^{y,-x_{j}}$. The new Hamiltonian in this basis of defects is

$$
H^{\prime}=-L-\sum_{i=1}^{L} \sigma_{i}^{z}+\sum_{i=1}^{L-1}\left(\sigma_{i}^{+} \sigma_{i+1}^{-}+\sigma_{i}^{-} \sigma_{i+1}^{+}+2 \sigma_{i}^{-} \sigma_{i+1}^{-}\right)
$$

This Hamiltonian shows clearly that the + spins are annihilated by pairs and, most importantly, cannot be created. Thus this Hamiltonian has a triangular character, which is a major simplification.

One can also check that all the events where a defect at site 1 hops to its left or one at site $L-1$ hops to its right have zero weight.

In this new basis the probabilities of the configurations of the defects are given as before by

$$
\left|P_{1}^{\prime}\right\rangle=e^{H^{\prime \prime}}\left|P_{0}^{\prime}\right\rangle
$$

and

$$
\rho_{L}(q)=\lim _{t \rightarrow \infty}\left\langle---\cdots-\left|\prod_{i=1}^{L}\left(1+\sigma_{i}^{-}\right) e^{H^{\prime} t}\right| P_{0}^{\prime}\right\rangle
$$




\subsection{The Fermions}

We can finally transform the Hamiltonian into a fermionic Hamiltonian by use of a Jordan-Wigner transformation. We define fermionic creation and annihilation operators as follows:

$$
\begin{aligned}
a_{i}^{+} & =(-)^{i-1} \sigma_{1}^{z} \cdots \sigma_{i-1}^{z} \sigma_{i}^{+} \\
a_{i} & =(-)^{i-1} \sigma_{1}^{z} \cdots \sigma_{i-1}^{z} \sigma_{i}^{-}
\end{aligned}
$$

Substituting these operators in (58) gives the following Hamiltonian:

$$
H^{\prime \prime}=-2 \sum_{i=1}^{L} a_{i}^{+} a_{i}+\sum_{i=1}^{L-1}\left(a_{i}^{+} a_{i+1}+a_{i+1}^{+} a_{i}-2 a_{i} a_{i+1}\right)
$$

Let us define the vacuum $|0\rangle$, which we identify with the state $|---\cdots--\rangle$ in the basis of the defects, by

$$
a_{i}|0\rangle=0 \quad \forall i
$$

and $\langle 0 \mid 0\rangle=1$. The vector $\left|P_{0}^{\prime}\right\rangle$ becomes, in terms of the fermionic operators,

$$
\left|P_{0}^{\prime \prime}\right\rangle=\left(\frac{1}{q}\right)^{L-1} \sum_{p} \sum_{x_{1}<y_{1}<x_{2}<y_{2} \ldots<y_{p}}\left(\prod_{j=1}^{p}(q-1)^{y_{j}-x_{j}}\right) a_{x_{1}}^{+} a_{y_{1}}^{+} \cdots a_{x_{p}}^{+} a_{y_{p}}^{+}|0\rangle
$$

One can perform a canonical transformation to eliminate the annihilating terms $a_{i} a_{i+1}$ in (60). For this, we define a set of annihilation and creation operators

$$
\begin{aligned}
b_{i} & =a_{i} \\
b_{i}^{+} & =a_{i}^{+}-\sum_{j \neq i} B_{i, j} a_{j}
\end{aligned}
$$

where $B_{i, j}=-B_{j, i}$ in order for (62) to define a canonical transformation. The matrix $B_{i, j}$ is defined for $1 \leqslant i, j \leqslant L$ and by convention we set $B_{0, i}=$ $B_{i, L+1}=0$. Expressing $(60)$ in terms of the $b_{i}, b_{i}^{+}$, we can eliminate the annihilating terms provided the $B_{i, j}$ obey

$$
4 B_{i, j}-B_{i-1, j}-B_{i+1, j}-B_{i, j-1}-B_{i, j+1}=2 \delta_{i+1, j} \quad \forall i<j
$$

which is identical to (44). The Hamiltonian (60) becomes

$$
H^{\prime \prime}=-2 \sum_{i=1}^{L} b_{i}^{+} b_{i}+\sum_{i=1}^{L-1}\left(b_{i}^{+} b_{i+1}+b_{i+1}^{+} b_{i}\right)
$$


The right and left eigenvectors of $(60)$ with zero eigenvalue are, respectively,

$$
|0\rangle \quad \text { and } \quad\langle 0| \exp \sum_{i<j} B_{i j} a_{j} a_{i}
$$

As

$$
p_{L}(q)=\lim _{t \rightarrow \infty}\left\langle 0\left|\left(1+a_{L}\right) \cdots\left(1+a_{1}\right) e^{t H^{\prime}}\right| P_{0}^{\prime \prime}\right\rangle
$$

and

$$
\lim _{i \rightarrow \infty} \exp \left(t H^{\prime}\right)=|0\rangle\langle 0| \exp \sum_{i<j} B_{i j} a_{j} a_{i}
$$

one obtains

$$
\begin{aligned}
\rho_{L}(q)= & \left(\frac{1}{q}\right)^{L-1} \sum_{p} \sum_{x_{1}<y_{1}<x_{2}<y_{2} \cdots<y_{p}}\left(\prod_{j=1}^{p}(q-1)^{y_{j}-x_{j}}\right) \\
& \times\left\langle 0\left|\left(\exp \sum_{i<j} B_{i j} a_{j} a_{i}\right) a_{x_{1}}^{+} a_{y_{1}}^{+} \cdots a_{x_{p}}^{+} a_{y_{p}}^{+}\right| 0\right\rangle
\end{aligned}
$$

Expanding the exponential, one ends up with (43).

\section{CONCLUSION}

In this paper we have derived an exact expression, ${ }^{(10),(29)-131)}$ valid at all times $t$ for the probability $r(q, t)$ that a spin never flips up to time $t$. This exact expression was complicated enough to make the calculation of the asymptotics and the determination of $\theta(q)$ rather difficult. We have recovered an expression identical to the one obtained in our previous work, ${ }^{(15)}$ where we used finite-size scaling arguments.

Surprisingly, in our approach, we had to separate the cases $q<2$ and $q>2$ and we obtained two different expressions (36) and (37) for $\theta(q)$, which turn out to be the analytic continuations of each other. This probably means that there are simpler ways of rewriting our expression for $r(q, t)$ where all the cases can be treated in the same manner. Unfortunately, we did not find these simpler expressions.

As $r(q, t)$ is known for all times $t$, one could try to extract other informations, ${ }^{(29)}$ in particular, the amplitude in front of the power law $t^{-\theta(q)}$ or other information on the reaction-diffusion problem in the presence of a fixed source. ${ }^{(30.31)}$

The approach used in this paper can be extended to calculate exactly the distribution of domain sizes for the zero-temperature dynamics of the 
1D Potts model. ${ }^{(32)}$ Also it seems possible ${ }^{(33)}$ to obtain some exact results on related reaction-diffusion models, such as the case of two species $A$ and $B$ with different diffusion constants. ${ }^{(34-36)}$

One can wonder whether, for the simple ID growth problem studied here, complicated exponents other than $\theta(q)$ could be defined and if the approach followed here could be of any use in calculating them.

Lastly, several unanswered questions remain on the extension to higher dimension: What is the upper critical dimension for $\theta(q)$ ? How can one measure the exponent $\theta(q)$ at finite temperature in $d>1$ ? What kind of approximate method can one imagine to predict $\theta(q)$ ?

\section{APPENDIX A}

In this appendix, we calculate the probability that, on the semiinfinite chain, $2 n$ walkers starting at the origin at times $t-\tau_{1}, t-\tau_{2}, \ldots, t-\tau_{2 n}$ do not meet up to time $t$.

The first part follows ref. 21 . If $p(x, t)$ is the probability that a walker starting at the origin at $t=0$ ends up at site $x$ at time $t$ [ the expression of $p(x, t)$ is given in (13) at the end of Section 2], then the probability $c_{i_{1}, i_{2}, \ldots, i_{2},}^{(n)}$ that no pair has met up to time $t$ is given by

$$
c_{i_{1}, i_{2}, \ldots, i_{2 n}}^{(n)}=\sum_{0 \leqslant x_{1} \leqslant x_{2} \leqslant \cdots x_{2 n}} \Delta\left(x_{1}, x_{2}, \ldots, x_{2 n} ; \tau_{1}, \tau_{2}, \ldots, \tau_{2 n}\right)
$$

where

$$
\Delta\left(x_{1}, x_{2}, \ldots, x_{2 n} ; \tau_{1}, \tau_{2}, \ldots, \tau_{2 n}\right)=\left|\begin{array}{cccc}
p\left(x_{1}, \tau_{1}\right) & p\left(x_{2}, \tau_{1}\right) & \cdots & p\left(x_{2 n}, \tau_{1}\right) \\
p\left(x_{1}, \tau_{2}\right) & p\left(x_{2}, \tau_{2}\right) & \cdots & p\left(x_{2 n}, \tau_{2}\right) \\
\vdots & \vdots & & \vdots \\
p\left(x_{1}, \tau_{2 n}\right) & p\left(x_{2}, \tau_{2 n}\right) & \cdots & p\left(x_{2 n}, \tau_{2 n}\right)
\end{array}\right|
$$

This is because the walkers which have not met remain in the same order and therefore can be treated as noninteracting fermions.

If $Q(x, t)$ is defined by

$$
Q(x, t)=\sum_{y \geqslant x} p(y, t)
$$

one finds by summing over the positions of the even walkers and by simplifying the determinant that

$$
c_{i_{1}, i_{2}, \ldots, i_{2 n}}^{(n)}=\sum_{0 \leqslant x_{1} \leqslant x_{3} \leqslant \ldots x_{2 n-1}} \Delta^{*}\left(x_{1}, x_{3}, \ldots, x_{2 n-1} ; \tau_{1}, \tau_{2}, \ldots, \tau_{2 n}\right)
$$

where 


$$
\begin{aligned}
& \Delta^{*}\left(x_{1}, x_{3}, \ldots, x_{2 n-1} ; \tau_{1}, \tau_{2}, \ldots, \tau_{2 n}\right) \\
& =\left|\begin{array}{ccccc}
p\left(x_{1}, \tau_{1}\right) & Q\left(x_{1}, \tau_{1}\right) & \cdots & p\left(x_{2 n-1}, \tau_{1}\right) & Q\left(x_{2 n-1}, \tau_{1}\right) \\
p\left(x_{1}, \tau_{2}\right) & Q\left(x_{1}, \tau_{2}\right) & \cdots & p\left(x_{2 n-1}, \tau_{1}\right) & Q\left(x_{2 n-1}, \tau_{2}\right) \\
\vdots & \vdots & & \vdots & \vdots \\
p\left(x_{1}, \tau_{2 n}\right) & Q\left(x_{1}, \tau_{2 n}\right) & \cdots & p\left(x_{2 n-1}, \tau_{2 n}\right) & Q\left(x_{2 n-1}, \tau_{2 n}\right)
\end{array}\right|
\end{aligned}
$$

Under this form, the determinant $\Delta^{*}$ is a symmetric function of the coordinates $x_{1}, x_{3}, \ldots, x_{2 n-1}$ and one can write

$$
c_{i_{1}, i_{2}, \ldots, i_{2 n}}^{(n)}=\frac{1}{n !} \sum_{0 \leqslant x_{1}} \sum_{0 \leqslant x_{3}} \ldots \sum_{0 \leqslant x_{2 n-1}} A^{*}\left(x_{1}, x_{3}, \ldots, x_{2 n-1} ; \tau_{1}, \tau_{2}, \ldots, \tau_{2 n}\right)
$$

Then by expanding the determinant, one ends up (see Appendix 7 of ref. 21) with the expression (12) for the $c^{(n)}$.

It is worth noticing that the $c^{(\prime)}$ give all the meeting probabilities between the walks. For example, if one considers four walks and if $c_{i, j}$ is the probability that the walks starting at times $t-\tau_{j}<t-t_{i}$ have not met up to time $t$, one has

$$
\begin{aligned}
& \psi_{1234}=1-c_{1,4} \\
& \psi_{123,4}=c_{1,4}-c_{1,3} \\
& \psi_{12,34}=c_{1,3}+c_{2,4}+c_{1,2,3,4}^{(2)}-c_{1,2}-c_{2,3}-c_{3,4} \\
& \psi_{1,234}=c_{1,4}-c_{2,4} \\
& \psi_{12,3,4}=c_{2,3}+c_{3,4}-c_{2,4}-c_{1,2,3,4}^{(2)} \\
& \psi_{1,23,4}=c_{1,3}+c_{2,4}-c_{2,3}-c_{1,4} \\
& \psi_{1,2,34}=c_{1,2}+c_{2,3}-c_{1,3}-c_{1,2,3,4}^{(2)} \\
& \psi_{1,2,3,4}=c_{1,2,3,4}^{(2)}
\end{aligned}
$$

where $\psi_{12.3 .4}$ is the probability that walkers 1 and 2 have met but walker 3 has neither met 2 nor 4 and similar definitions hold for the other $\psi$. It is easy to check the validity of (A1): for example,

$$
\begin{aligned}
& c_{1,2}=\psi_{1,234}+\psi_{1,2,34}+\psi_{1,23,4}+\psi_{1,2,3,4} \\
& c_{1,3}=\psi_{1,234}+\psi_{12,34}+\psi_{1,23,4}+\psi_{1,2,34}+\psi_{12,3,4}+\psi_{1,2,3,4}
\end{aligned}
$$

This example shows that once the $c^{(n)}$ are known, the probability $P(m, t)$ of finding $m$ walkers at time $t$ can be determined. In the case of four walks one finds 


$$
\begin{aligned}
& P(1, t)=\psi_{1234}=1-c_{1,4} \\
& P(2, t)=\psi_{123,4}+\psi_{12,34}+\psi_{1,234}=2 c_{1,4}-c_{1,2}-c_{2,3}-c_{3,4}+c_{1,2,3,4}^{(2)} \\
& P(3, t)=\psi_{12,3,4}+\psi_{1,23,4}+\psi_{1,2,34}=c_{1,2}+c_{2,3}+c_{3,4}-c_{1,4}-2 c_{1,2,3,4}^{(2)} \\
& P(4, t)=\psi_{1,2,3,4}=c_{1,2,3,4}^{(2)}
\end{aligned}
$$

More generally, if one has $N$ walkers, there are $2^{N-1}$ possible $\psi$, and $N(N-1) / 2$ elements $c_{i, j}, N(N-1)(N-2)(N-3) / 24$ elements $c_{i, j, k, l}^{(2)}, \ldots$ There are therefore all together $2^{N-1}-1$ parameters $c$ which determine all the $\psi$ (there is one more $\psi$ than $c$, which is fixed by the fact that the sum of all the $\psi$ is 1$)$.

\section{APPENDIX B}

In this appendix, we show how sums such as (18), (24), and (43) can be expressed as determinants. A convenient way is provided by the following identity: given two antisymmetric $N \times N$ matrices $a$ and $c$, one has

$$
\begin{aligned}
\operatorname{det}(I+a c)= & {\left[1-\sum_{i<j} a_{i, j} c_{i, j}+\sum_{i<j<k<1} a_{i, j, k, 1}^{(2)} c_{i, j, k, l}^{(2)}+\cdots\right.} \\
& \left.+(-1)^{n} \sum_{i_{1}<i_{2}<\cdots<i_{2 n}} a_{i_{1}, i_{2}, \ldots, i_{2 n-1}, i_{2 n}}^{(n)} c_{i_{1}, i_{2}, \ldots, i_{2 n-1, i_{2 n}}}^{(n)}+\cdots\right]^{2}
\end{aligned}
$$

where $a_{i_{1}, i_{2} \ldots, i_{2 n-1}, i_{2 n}}^{(n)}$ and $c_{i_{1}, i_{2} \ldots \ldots i_{2 n-1}, i_{2 n}}^{(n)}$ are the Pfaffians of the matrices $a$ and $c$ defined in Eqs. (11) and (12). Before giving a proof of (B1), we explain how various relations used in the main text follow from it.

\section{B1. Equivalence Between (18) and (21)}

Let us first choose $a$,

$$
a=\mu\left(\begin{array}{rrrrrr}
0 & 1 & 0 & & 0 & \lambda \\
-1 & 0 & 1 & 0 & & 0 \\
0 & -1 & 0 & 1 & 0 & \\
& 0 & . & 0 & . & \\
0 & & & . & & 1 \\
-\lambda & 0 & & & -1 & 0
\end{array}\right)
$$


Of all the possible contractions contributing to the Pfaffian of order $n$, only two give a nonvanishing result. Therefore

$$
\begin{aligned}
a_{i_{1}, i_{2} \ldots, i_{2 n-1}, i_{2 n} n}^{(n)}= & \mu^{n}\left(\delta_{i_{1}+1 . i_{2}} \cdots \delta_{i_{2 n-1}+1 . i_{2 n}}\right. \\
& \left.+\lambda \delta_{i_{1}, 1} \delta_{i_{2}+1, i_{3}} \cdots \delta_{i_{2 n-2}+1 . i_{2 n-1}} \delta_{i_{2 n}, N}\right)
\end{aligned}
$$

So, with the choice (B2) for $a$, (B1) simply becomes

$$
\begin{aligned}
\operatorname{det}(I+a c)= & {\left[1-\mu \sum_{i=1}^{N-1} c_{i, i+1}+\mu^{2} \sum_{i<j} c_{i, i+1, j, j+1}^{(2)}-\cdots\right.} \\
& \left.-\lambda\left\{\mu c_{1, N}-\mu^{2} \sum_{i} c_{1, i, i+1, N}^{(2)}+\cdots\right\}\right]^{2}
\end{aligned}
$$

and as (18) is positive, it can be rewritten as (21).

\section{B2. Derivation of $(24),(25)$}

It is also possible to express $A_{N}\left(\tau_{1}, \ldots, \tau_{N}\right)$ using instead of $c$ the antisymmetric matrix $b$ such that $c_{i, j}=1-b_{i, j}$ for $i<j$. That is, $c=b^{\prime}-b$ with $b_{i, j}^{\prime}=-b_{j, i}^{\prime}=1$ for $i<j$. The determinant can be rewritten as

$$
\begin{aligned}
\operatorname{det}[I+a c] & =\operatorname{det}\left[I+a b^{\prime}-a b\right] \\
& =\operatorname{det}\left[I+a b^{\prime}\right] \operatorname{det}\left[I-\left(I+a b^{\prime}\right)^{-1} a b\right]
\end{aligned}
$$

All the Pfaffians of $b^{\prime}$ are 1 . Therefore, using ( $\mathrm{B} 1$ ), one finds

$$
\begin{aligned}
\operatorname{det}\left[I+a b^{\prime}\right]= & {\left[1-(N-1) \mu+\frac{(N-2)(N-3)}{2} \mu^{2}+\cdots\right.} \\
& -\lambda\{1-(N-2) \mu+\cdots\}]^{2}
\end{aligned}
$$

and this gives, using (19), (20),

$$
\sqrt{\operatorname{det}\left[I+a b^{\prime}\right]}=q^{-(N-1)}
$$

One can also check that $-\left(I+a b^{\prime}\right)^{-1} a$ is equal to the antisymmetric matrix $A$ such that

$$
\Lambda_{i, j}=-\lambda^{j-i}
$$


for $i<j$ (for example, by multiplying $A$ on the left by $I+a b^{\prime}$ ). Finally, (B5) gives the alternative expression of $A_{N}\left(\tau_{1}, \ldots, \tau_{N}\right)$ as

$$
A_{N}\left(\tau_{1}, \ldots, \tau_{N}\right)=\frac{1}{q^{N-1}} \sqrt{\operatorname{det}(I+A b)}
$$

This determinant can also be expanded as a sum of Pfaffians of the matrix $b$. Since $A_{i, j}$ is of the form $-f(j) / f(i)$, one has a simple expression for the Pfaffian to order $n$ :

$$
A_{i_{1}, i_{2}, \ldots, i_{2 n-1}, i_{2 n}}^{(n)}=A_{i_{1}, i_{2}} \cdots \Lambda_{i_{2 n-1}, i_{2 n}}=(-1)^{n} \lambda^{\left(-i_{1}+i_{2}+\cdots-i_{2 n-1}+i_{2 n}\right)}
$$

and using the identity (B1), one obtains

$$
\begin{aligned}
A_{N}\left(\tau_{1}, \ldots, \tau_{N}\right)= & \frac{1}{q^{N-1}}\left\{1+\sum_{i<j}(q-1)^{j-i} b_{i, j}\right. \\
& \left.+\sum_{i<j<k<1}(q-1)^{I-k+j-i} b_{i, j, k .1}^{(2)}+\cdots\right\}
\end{aligned}
$$

This completes the proof that (18), (21), (24), and (25) are equivalent.

\section{B3. Proof of (B1)}

Equation (B1) can be obtained by computing in two ways det $M$, where $M$ is the $2 N \times 2 N$ antisymmetric matrix

$$
M=\left(\begin{array}{cc}
a & -I \\
I & c
\end{array}\right)
$$

On the one hand, one can show that $\operatorname{det} M=\operatorname{det}(I+a c)$ by computing, for example, the determinant on both sides of

$$
\left(\begin{array}{cc}
a & -I \\
I & c
\end{array}\right)\left(\begin{array}{cc}
c & I \\
-I & a
\end{array}\right)=\left(\begin{array}{cc}
I+a c & 0 \\
0 & I+c a
\end{array}\right)
$$

On the other hand, since $M$ is antisymmetric, its determinant is the square of a Pfaffian'21):

$$
\operatorname{det} M=\left[\frac{1}{2^{N} \cdot N !} \sum_{\sigma} \varepsilon(\sigma) M_{i_{\sigma 11}, i_{\sigma(2)}} \cdots M_{i_{\sigma\left(2 N-11, i_{\sigma 22 N}\right.}}\right]^{2}
$$


Ordering the terms of the r.h.s. of (B12) in increasing powers of the matrix elements of $a$ and $c$ gives

$$
\begin{aligned}
\operatorname{det} M= & {\left[1-\sum_{i<j} a_{i, j} c_{i, j}+\sum_{i<j<k<1} a_{i, j, k, 1}^{(2)} c_{i, j, k, 1}^{(2)}+\cdots\right.} \\
& \left.+(-1)^{n} \sum_{i_{1}<i_{2}<\cdots<i_{2 n}} a_{i_{1}, i_{2}, \ldots, i_{2 n-1}, i_{2 n}}^{(n)} c_{i_{1}, i_{2}, \ldots, i_{2 n-1, i_{2 n}}}^{(n)}+\cdots\right]^{2}
\end{aligned}
$$

[the first term corresponds to all permutations where $i$ is paired with $i+N$ for all indices $1 \leqslant i \leqslant N$, the second to permutations where the same is true except for one couple of indices $i, j(1 \leqslant i \leqslant N, 1 \leqslant j \leqslant N)$, where instead $i$ is paired with $j$ and $i+N$ is paired with $j+N, \ldots]$.

\section{APPENDIX C}

Consider a matrix $c\left(t, t^{\prime}\right)$ having the following properties:

$$
\begin{aligned}
& c\left(t, t^{\prime}\right)=-c\left(t^{\prime}, t\right)>0 \quad \text { if } t<t^{\prime} \\
& c\left(t, t^{\prime}\right) \rightarrow 1 \quad \text { for } t^{\prime} \rightarrow \infty \\
& c(t, t)=0
\end{aligned}
$$

The first property implies that

$$
\frac{d}{d t} c\left(\tau_{1}, \tau_{2}\right)=-\frac{d}{d t^{\prime}} c\left(\tau_{2}, \tau_{1}\right)
$$

( $d / d t$ is the derivative with respect to the first variable and $d / d t^{\prime}$ is the derivative with respect to the second variable). Let us define

$$
\begin{aligned}
& u_{n}=\int_{a}^{b} d \tau_{1} \int_{a}^{b} d \tau_{2} \cdots \int_{a}^{b} d \tau_{n} c\left(a, \tau_{1}\right) \frac{d}{d t} c\left(\tau_{1}, \tau_{2}\right) \cdots \frac{d}{d t} c\left(\tau_{n}, b\right) \\
& v_{n}=\int_{a}^{b} d \tau_{1} \int_{a}^{b} d \tau_{2} \cdots \int_{a}^{b} d \tau_{n} c\left(b, \tau_{1}\right) \frac{d}{d t} c\left(\tau_{1}, \tau_{2}\right) \cdots \frac{d}{d t} c\left(\tau_{n}, b\right) \\
& x_{n}=\int_{a}^{b} d \tau_{1} \int_{a}^{b} d \tau_{2} \cdots \int_{a}^{b} d \tau_{n} c\left(a, \tau_{1}\right) \frac{d}{d t} c\left(\tau_{1}, \tau_{2}\right) \cdots \frac{d}{d t} c\left(\tau_{n}, a\right) \\
& y_{n}=\int_{a}^{b} d \tau_{1} \int_{a}^{b} d \tau_{2} \cdots \int_{a}^{b} d \tau_{n} c\left(b, \tau_{1}\right) \frac{d}{d t} c\left(\tau_{1}, \tau_{2}\right) \cdots \frac{d}{d t} c\left(\tau_{n}, a\right)
\end{aligned}
$$


[for $n=0$, we define $u_{0}=-y_{0}=c(a, b)$ and $v_{0}=x_{0}=0$ ]. One can show, using integration by parts, that

$$
\begin{aligned}
u_{1} & =-y_{1} \\
u_{2} & =-y_{2}+x_{1} u_{0}-y_{0} v_{1} \\
& \vdots \\
u_{n} & =-y_{n}+\sum_{i=1}^{n-1}\left(x_{i} u_{n-i-1}-v_{i} y_{n-i-1}\right)
\end{aligned}
$$

One can also show that

$$
\begin{aligned}
v_{1} & =\frac{1}{2} c^{2}(a, b) \\
v_{2} & =c(a, b) u_{1} \\
v_{3} & =c(a, b) u_{2}+\frac{1}{2}\left(u_{1}^{2}-v_{1}^{2}\right) \\
& \vdots \\
v_{n} & =c(a, b) u_{n-1}+\frac{1}{2}\left[\sum_{i=1}^{n-2}\left(u_{i} u_{n-i-1}-v_{i} v_{n-i-1}\right)\right]
\end{aligned}
$$

Similarly, one can show that

$$
\begin{aligned}
x_{1} & =-\frac{1}{2} c^{2}(a, b) \\
x_{2} & =c(a, b) y_{1} \\
& \vdots \\
x_{n} & =c(a, b) y_{n-1}+\frac{1}{2}\left[\sum_{i=1}^{n-2}\left(x_{i} x_{n-i-1}-y_{i} y_{n-i-1}\right)\right]
\end{aligned}
$$

Clearly these recursion relations imply that

$$
y_{n}=-u_{n} ; \quad x_{n}=-v_{n}
$$

so that the knowledge of the sequence $u_{n}$ determines all the rest.

The matrix elements

$$
\begin{aligned}
& \left(c M^{-1}\right)_{a, b}=\sum_{n=0}^{\infty}(-2 \mu)^{\prime \prime} u_{n} \\
& \left(c M^{-1}\right)_{b, b}=\sum_{n=0}^{\infty}(-2 \mu)^{n} v_{n}
\end{aligned}
$$




$$
\begin{aligned}
& \left(c M^{-1}\right)_{a, a}=\sum_{n=0}^{\infty}(-2 \mu)^{n} x_{n} \\
& \left(c M^{-1}\right)_{b, a}=\sum_{n=0}^{\infty}(-2 \mu)^{n} y_{n}
\end{aligned}
$$

The above relations imply that

$$
\begin{aligned}
& \left(c M^{-1}\right)_{a, b}=-\left(c M^{-1}\right)_{b, a} \\
& \left(c M^{-1}\right)_{b, b}=-\left(c M^{-1}\right)_{a, a} \\
& \left(c M^{-1}\right)_{b, b}=\mu\left\{\left[\left(c M^{-1}\right)_{b, b}\right]^{2}-\left[\left(c M^{-1}\right)_{a, b}\right]^{2}\right\}
\end{aligned}
$$

So, if $\left(c M^{-1}\right)_{a, b}$ is known, the other three matrix elements follow easily.

\section{C1. Asymptotics}

When $b$ is large (with $a$ fixed so that the ratio $b / a$ is large), $(d / d t) c\left(\tau, \tau^{\prime}\right)$ is nonzero only when the ratio $\tau / \tau^{\prime}$ is of order 1 . Consequently, $u_{n}$ can be replaced by

$$
u_{n}=\int_{a}^{b} d \tau_{1} \int_{a}^{b} d \tau_{2} \cdots \int_{a}^{b} d \tau_{n} \frac{d}{d t} c\left(\tau_{1}, \tau_{2}\right) \cdots \frac{d}{d t} c\left(\tau_{n}, b\right)
$$

because $b / \tau_{n}$ is of order $1, \tau_{n} / \tau_{n-1}$ is of order 1 , and so on, so that $b / \tau_{1}$ is of order 1 and so $c\left(a, \tau_{1}\right) \simeq 1$. This implies that

$$
u_{n} \simeq v_{n-1}-u_{n-1}
$$

and therefore

$$
\left(c M^{-1}\right)_{a, b} \simeq 1-2 \mu\left[\left(c M^{-1}\right)_{b, b}-\left(c M^{-1}\right)_{a, b}\right]
$$

and this together with the previous relations $(\mathrm{Cl})-(\mathrm{C} 3)$ implies that for $b / a \rightarrow \infty$,

$$
\begin{aligned}
& \left(c M^{-1}\right)_{a, b}=-\left(c M^{-1}\right)_{b, a} \simeq \frac{1}{\sqrt{1-4 \mu}} \\
& \left(c M^{-1}\right)_{b, b}=-\left(c M^{-1}\right)_{a, a} \simeq \frac{1}{2 \mu}-\frac{1-2 \mu}{2 \mu \sqrt{1-4 \mu}}
\end{aligned}
$$




\section{APPENDIX D}

In this appendix we show how the reaction-diffusion problem discussed in Section 6 of coalescing particles on a finite system of $L$ sites with a fixed source at the origin $(0=L)$ can be reduced to the Hamiltonian (53)-(55).

Let us call $P_{1}\left(x_{1}, \ldots, x_{m-1}\right)=P_{1}\left(\left\{x^{m}\right\}\right)$ the probability of finding, at time $t$, particles at positions $1 \leqslant x_{1}<\cdots<x_{m-1} \leqslant L-1$. All the other sites are empty except the source at position $0(=L)$, which is always occupied, and the total number of particles $m$ can take all the possible values from 1 to $L$. The $P_{r}\left(\left\{x^{m}\right\}\right)$ represent exclusive probabilities; thus they are positive and their sum is one: $\sum_{n} \sum_{\left\{x^{m}\right\}} P\left(\left\{x^{m}\right\}\right)=1$. We consider the state

$$
\left|P_{1}\right\rangle=\sum_{m} \sum_{\left\{x^{m}\right\}} P_{r}\left(\left\{x^{\prime \prime \prime}\right\}\right)|(+)-+--+\cdots\rangle
$$

where each occupied site corresponds to a + and each empty site to a The + spin in parentheses represents the site 0 , which is always occupied.

The time evolution of $\left|P_{1}\right\rangle$ is given by

$$
\frac{d}{d t}\left|P_{t}\right\rangle=H\left|P_{t}\right\rangle
$$

with $H$ is a spin-chain Hamiltonian

$$
H=g_{1}+h_{1,2}+\cdots+h_{L-2, L-1}+g_{L-1}
$$

with

$$
g_{1}=\sigma_{1}^{+}+\sigma_{1}^{-}-1 ; \quad g_{L-1}=\sigma_{L-1}^{+}+\sigma_{L-1}^{-}-1
$$

and

$$
\begin{aligned}
h_{i, i+1}= & \sigma_{i}^{-} \sigma_{i+1}^{+}+\sigma_{i}^{+} \sigma_{i+1}^{-}+\frac{1}{2} \sigma_{i}^{-} \sigma_{i+1}^{z} \\
& +\frac{1}{2} \sigma_{i}^{z} \sigma_{i+1}^{-}+\frac{1}{2} \sigma_{i}^{-}+\frac{1}{2} \sigma_{i+1}^{-}-\frac{1}{2} \sigma_{i}^{z}-\frac{1}{2} \sigma_{i+1}^{z}-1
\end{aligned}
$$

so that

$$
\begin{aligned}
\dot{H}= & -L+\sigma_{1}^{+}+\sigma_{1}^{-}+\sigma_{L-1}^{+}+\sigma_{L-1}^{-} \\
& +\frac{1}{2} \sum_{i=1}^{L-2} 2 \sigma_{i}^{-} \sigma_{i+1}^{+}+2 \sigma_{i}^{+} \sigma_{i+1}^{-}+\sigma_{i}^{-} \sigma_{i+1}^{z}+\sigma_{i}^{z} \sigma_{i+1}^{-} \\
& +\sigma_{i}^{-}+\sigma_{i+1}^{-}-\sigma_{i}^{z}-\sigma_{i+1}^{-}
\end{aligned}
$$




\section{D1. A Change of Basis}

Let us make the following transformation on $H$ :

$$
H^{\prime}=U^{-1} H U
$$

where

$$
U=\prod_{i=1}^{L-1}\left(1-\sigma_{i}^{+}\right) ; \quad U^{-1}=\prod_{i=1}^{L-1}\left(1+\sigma_{i}^{+}\right)
$$

In the new basis, the steady state is given by $\left|P_{\infty}^{\prime}\right\rangle=U^{-1}\left|P_{\infty}\right\rangle$ and satisfies

$$
H^{\prime}\left|P^{\prime}\right\rangle=0
$$

The new Hamiltonian $H^{\prime}$ takes the form

$$
H^{\prime}=g_{1}^{\prime}+h_{1,2}^{\prime}+\cdots+h_{L-2, L-1}^{\prime}+g_{L-1}^{\prime}
$$

where

$$
g_{1}^{\prime}=\sigma_{1}^{-}+\sigma_{1}^{\overline{1}}-1 ; \quad g_{L-1}^{\prime}=\sigma_{L-1}^{-}+\sigma_{L-1}^{z}-1
$$

and

$$
\begin{aligned}
h_{i, i+1}^{\prime}= & -1+\frac{1}{2}\left[\sigma_{i}^{-} \sigma_{i+1}^{\bar{z}}+\sigma_{i}^{z} \sigma_{i+1}^{-}-\sigma_{i}^{+} \sigma_{i+1}^{z}-\sigma_{i}^{z} \sigma_{i+1}^{+}\right. \\
& \left.+2 \sigma_{i}^{z} \sigma_{i+1}^{z}+\sigma_{i}^{-}+\sigma_{i+1}^{-}+\sigma_{i}^{+}+\sigma_{i+1}^{+}\right]
\end{aligned}
$$

This is, up to a transposition, the Hamiltonian (53)-(55) of Section 7.

\section{REFERENCES}

1. A. J. Bray, Adv. Phys. 43:357 (1994).

2. R. J. Glauber, J. Math. Phys. 4:294 (1963).

3. T. M. Liggett, Interacting Particle Systems (Springer-Verlag, New York, 1985).

4. Z. Rácz, Phys. Rev. Lett. 55:1707 (1985).

5. A. J. Bray, J. Phys. A 23:L67 (1990).

6. J. G. Amar and F. Family, Phys. Rev. A 41:3258 (1990).

7. B. Derrida, C. Godrèche, and I. Yekutieli, Phys. Rev. A 44:6241 (1991).

8. V. Privman, J. Stat. Phys. 69:629 (1992).

9. H. Furukuwa, Phys. Rev. B 40:2341 (1989).

10. D. S. Fisher and D. A. Huse, Phys. Rev. B 38:373 (1988).

11. T. J. Newman, A. J. Bray, and M. A. Moore. Phys. Rev. B 42:4514 (1990).

12. B. Derrida, A. J. Bray, and C. Godrèche, J. Phys. A 27:L357 (1994).

13. D. Stauffer, J. Phys. A 27:5029 (1994).

14. B. Derrida, J. Phys. A 28:1481 (1995). 
15. B. Derrida, V. Hakim, and V. Pasquier, Phys. Rev. Lett. 75:751 (1995).

16. F. Leyvraz and S. Redner, Phys. Rev. A 46:3132 (1992).

17. S. Cornell and M. Droz, Phys. Rev. Lett. 70:3824 (1993).

18. K. Kang and S. Redner, Phys. Rev. Lett. 52:955 (1984).

19. S. Cornell, M. Droz, and B. Chopard, Physica A 188:322 (1992).

20. F. C. Alcaraz, M. Droz, M. Henkel, and V. Rittenberg, Ann. Phys. 230:250 (1994).

21. M. L. Mehta, Random Matrices (Academic Press, New York, 1967).

22. G. Szegö, Commun. Sém. Math. Univ. Lund 228 (1952).

23. M. Kac, Duke Math. J. 21:501 (1954).

24. B. M. McCoy and T. T. Wu, The Two-Dimensional Ising Model (Harvard University Press, Cambridge, Massachusetts, 1973), and references therein.

25. M. Bramson and D. Griffeath, Ann. Prob. 8:183 (1980).

26. J. L. Spouge, Phys. Rev. Lett. 60:871 (1988).

27. D. ben-Avraham, M. A. Burschka, and C. R. Doering, J. Stat. Phys. 60:695 (1990).

28. K. Krebs, M. P. Pfanmnmüller, B. Wehefritz, and H. Hinrichsen, J. Stat. Phys. 78:1429 (1995).

29. E. Ben-Naim, L. Frachebourg, and P. L. Krapivsky, Plyss. Rev. E 53:3078 (1996).

30. Z. Cheng, S. Redner, and F. Leyvraz, Phys. Rev. Lett. 62:2321 (1989).

31. P. L. Krapivsky, Physica A 198:157 (1993).

32. B. Derrida and R. Zeitak, preprint 96, submitted to Phys. Rev. E.

33. C. Monthus, Work in progress.

34. P. L. Krapivsky, E. Ben-Naim, and S. Redner, Phys. Rev. E 50:2474 (1994).

35. J. L. Cardy, J. Phys. A 28:L19 (1995).

36. M. Howard, Preprint (1995).

37. C. Itzykson and J. M. Drouffe, Staristical Field Theory, Vol. 1 (Cambridge University Press. Cambridge. 1989). 